Article

\title{
Chemometric Characterization of Strawberries and Blueberries according to Their Phenolic Profile: Combined Effect of Cultivar and Cultivation System
}

\author{
Milica Fotirić Akšić ${ }^{1, *}$, Dragana Dabić Zagorac ${ }^{2}$, Milica Sredojević ${ }^{2}{ }^{\circledR}$, Jasminka Milivojević ${ }^{1}$, \\ Uroš Gašić $^{3}{ }^{\circledR}$, Mekjell Meland ${ }^{4}$ and Maja Natić ${ }^{5}$ \\ 1 Faculty of Agriculture, University of Belgrade, 11080 Belgrade, Serbia; jasminka@agrif.bg.ac.rs \\ 2 Innovation Center, University of Belgrade-Faculty of Chemistry, P.O. Box 51, 11158 Belgrade, Serbia; \\ ddabic@chem.bg.ac.rs (D.D.Z.); pantelicm@chem.bg.ac.rs (M.S.) \\ 3 Institute for Biological Research "Siniša Stanković" - National Institute of Republic of Serbia, University of \\ Belgrade, 11060 Belgrade, Serbia; uros.gasic@ibiss.bg.ac.rs \\ 4 Norwegian Institute of Bioeconomy Research-NIBIO Ullensvang, NO-5781 Lofthus, Norway; \\ mekjell.meland@nibio.no \\ 5 Faculty of Chemistry, University of Belgrade, P.O. Box 51, 11158 Belgrade, Serbia; mmandic@chem.bg.ac.rs \\ * Correspondence: fotiric@agrif.bg.ac.rs; Tel.: +381-64-2612710
}

Academic Editor: Derek J. McPhee

Received: 30 October 2019; Accepted: 24 November 2019; Published: 26 November 2019

\begin{abstract}
Chemical characterizations of leaves and fruits that were obtained from organically and integrally produced strawberries ('Favette', 'Alba', and 'Clery') and blueberries ('Bluecrop', 'Duke', and ${ }^{\prime}{ }^{N u i}$ ') from western Serbia were undertaken in this study. Phenolic analysis was done while using ultra-high performance liquid chromatography coupled to a linear ion trap-Orbitrap hybrid mass analyzer, while total phenolic content (TPC), total anthocyanin content (TAC), and radical-scavenging activity (RSA) by spectrophotometry. In general, leaves and fruits from blueberry showed higher levels of TPC and TAC as compared to strawberry. These chemical traits were larger in organic grown fruits and larger in leaves than fruits. The most abundant phenolics in leaves and fruits of blueberry was 5-O-caffeoylquinic acid, followed by quercetin 3-O-galactoside, while catechin, quercetin, and kaempferol 3-O-glucosid were dominant in the leaves and fruits of strawberry. cis, trans-Abscisic acid was detected in all fruit samples, but not in leaves. Blueberries (both fruits and leaves) were separated from strawberries, but only organic blueberry fruits were distinguished from integrated fruits, according to principal component analysis. Quercetin, kaempferol, 5-O-caffeoylquinic acid, ferulic acid, caffeic acid, catechin, $p$-coumaric acid, and $p$-hydroxybenzoic acid were the most influential phenolic compounds for the separation. Much higher contents of TPC, RSA, TAC, quercetin 3-O-galactoside, and quercetin were found in fruits and TPC, RSA, catechin, $p$-hydroxybenzoicacid, $p$-coumaricacid, and ferulic acid in leaves in all three blueberry cultivars and the strawberry cultivar 'Clery'. These phenolic compounds are good sources of antioxidant compounds with potentially high beneficial effects on human health.
\end{abstract}

Keywords: Fragaria $\mathrm{x}$ ananassa; Vaccinium corymbosum; organic production; integrated production; fruit; leaf; phenolic profiles; antohocyanin; principal component analysis

\section{Introduction}

Cultivated strawberry (Fragaria $x$ ananassa Duch.) and highbush blueberry (Vaccinium corymbosum L.) are considered to be economically important freshly consumed fruits, and the production volumes are rapidly increasing worldwide during recent years [1-4]. Organic and integrated productions 
are two most dominant fruit productions in Europe. Integrated production follows Integrated Pest Management Directive 2009/128/ EC, while organic production is implementing European Action Plan for Organic Food and Farming [Council Regulation (EC) 834/2007]. Some principles of both production systems are similar and both products are considered to be 'premium' food, but as compared to organic production, where no synthetic pesticides and fertilizers are allowed, in integrated production the use of chemicals and artificial inputs are not restricted [5].

Lately, there has been an increasing interest in organic fruit production due to environmental, economic, and social concerns. Besides avoiding chemicals, it lowers soil degradation, minimizes non-recyclable inputs and the presence of pesticide residues in food, manages animals extensively with focus on their wellbeing, and preserves natural resources and the rural landscapes, thus making it environmentally friendlier, safer, and contributing to the global food security [6,7]. Berry-fruits are the fastest growing organic fruit category, with an increased area of nearly $300 \%$ in the last decade worldwide [8]. In 2017, organic berry productions were grown on 63,543 ha, with Poland, Chile, and Spain as the leading countries [9]. Some scientific studies affirmed the nutritional value of organic fruits based on their greater concentration of particularly potent phenolic compounds that are believed to be more nutritious and beneficial to human health [10-12].

Besides their exquisite flavor, berry-fruits have gained significant attention by consumers due to a high content of health promoting compounds based on considerable quantities of different phytochemicals. Strawberries and blueberries are both relevant sources of phenolic compounds, including both flavonoid and non-flavonoid types, which mainly represent anthocyanins, flavonols, and flavanols, followed by phenolic acids (hydroxybenzoic/hydroxycinnamic acids), as well as hydrolysable (ellagitannins and gallotannins) and some condensed tannins [4,13-15]. These berry-fruit phenolics are well known for their antioxidant, anti-inflammatory, antimicrobial, antihypertensive, anti-allergy, and antidiabetic properties [11,16-18]. Other plant parts can also have medical application and they have been used in various forms [19]. Leaves contain many bioactive compounds, including flavonoids, ascorbic acid, tannins, and essential oils, which also act as powerful antioxidants that neutralize the harmful effects that are associated with reactive oxygen species [20]. Blueberry leaves are found to be a prospective source of phenolic compounds, such as anthocyanins and chlorogenic acid, ranging from 2.321-2.636 mg of malvidin 3-glucoside/g and from 49.34-52.66 mg of chlorogenic acid/g dry matter, respectively [21]. Leaves from the Vaccinium species show a liver lipid-lowering effect [22], and present neuroprotective [23], hypotensive [24], and anti-diabetic activity [25]. Potentially health-promoting phenolic compounds have also been found in strawberry leaves (including gallic acid derivatives, ellagitannins, chlorogenic acids, flavonoids, and proanthocyanidins). The leaf extract of strawberries can be used to treat diabetes nephropathy by regulating thyroid hormones, which play an important role in the metabolism of glucose and lipids [26,27].

Quantitative variations in phenolic compounds are mostly associated with genetic background, particularly in its interaction with the environmental factors, cultivation systems, and growing location [2,14,28-33]. It can also differ in certain stages of plant development and fruit ripeness [3,15,34,35], showing non-uniform concentrations in all parts of the plant [36,37]. Huge variability has been recorded among different strawberry and blueberry cultivars in terms of phenolic content and, correspondingly, the expressed total antioxidant capacity [31,38,39]. Cultivation techniques can additionally influence the phytochemical content of strawberries $[4,30,40]$ and blueberries $[3,41,42]$. In that regard, growing system and agricultural practices should be adjusted to the needs of a single cultivar or group of cultivars with similar requirements to enhance their bioactive potential [43].

Few studies on nutritional fruit quality contrasting organic with conventionally/integrated grown fruits are conducted with a focus on differences in phenolic composition related to the cultural systems $[7,42,44]$. Therefore, the main objective of this study was to evaluate how organic and integrated farming affect quantitative variations in individual phenolic compounds and their distribution in fruits and leaves, as well as the total phenolic content and corresponding antioxidant capacity of three strawberry and three blueberry cultivars. 


\section{Results and Discussion}

2.1. Total Phenolic Content (TPC), Radical-Scavenging Activity (RSA), and Total Anthocyanin Content (TAC) Results

Some differences were noticeable between blueberry and strawberry fruits when comparing the results that are presented in Table 1 . Higher contents of total phenolics and total anthocyanins were observed in blueberry fruit samples (TPC: 2.27-6.26 g GAE/kg; TAC: 0.62-2.86 g cy-3-glu/kg) than in strawberry fruits (TPC: $1.18-2.27 \mathrm{~g} \mathrm{GAE} / \mathrm{kg}$; TAC: $0.16-0.37 \mathrm{~g}$ cy-3-glu/kg). These results are in line with the database URL:http://www.phenol-explorer.eu, where the TPC of high bush blueberry and strawberry fruits ranged from 0.2 to $8.68 \mathrm{~g} \mathrm{GAE} / \mathrm{kg}$ FW (based on nine references) and from 0.72 to $4.43 \mathrm{~g} \mathrm{GAE} / \mathrm{kg}$ FW (based on seven references), respectively. Interestingly, the ranges that were obtained for radical-scavenging activities in blueberries and strawberries were both similar (18.31-33.83 mmol TE/kg and 16.32-24.05 mmol TE/kg). A bit lower ranges for all three traits (TPC, RSA, and TAC) were obtained by Panico et al. [45] for two strawberry cultivars (Tudla and Maletto) and for TAC and RSA by Crespo et al. [46] for cultivars 'Antea', 'Asis', and 'Matis'. This is probably due to the different cultivars and agro-climatic conditions tested. All of the organically produced strawberry and blueberry fruit samples were characterised with significantly higher RSA, TPC, and TAC values when compared with integrated fruits (with the exception of strawberry Favette' where the opposite trend was observed). This is in accordance with Ochmian et al. [7], who worked with blueberries and Olsson et al. [11], who studied strawberries, and proved that both organically grown fruit species have a higher level of TPC and TAC when compared to integrally produced fruits. This could be due to the fact that organically maintained soils have more total carbon and micronutrients, together with a greater microbial activity, which all together affect plant metabolism and biosynthesis of compounds with antioxidant activity [47].

In blueberry fruits, the highest values for TPC, RSA, and TAC were determined in organic 'Duke' (6.26 g GAE/kg, $33.83 \mathrm{mmol} \mathrm{TE} / \mathrm{kg}$, and $2.86 \mathrm{~g}$ cy-3-glu/kg, respectively). Generally, TPC in blueberries ranged from $2.27 \mathrm{~g} \mathrm{GAE} / \mathrm{kg}$ (integrated 'Bluecrop') to $4.38 \mathrm{~g}$ GAE/kg (organic 'Bluecrop'), RSA was in the range from $18.31 \mathrm{mmol} \mathrm{TE} / \mathrm{kg}$ (integrated 'Nui') to $25.36 \mathrm{mmol} \mathrm{TE} / \mathrm{kg}$ (integrated 'Duke'), while the range of TAC was between $0.62 \mathrm{~g}$ cy-3-glu/kg (integrated 'Bluecrop') and $1.80 \mathrm{~g} \mathrm{cy-3-glu/kg} \mathrm{(integrated} \mathrm{'Duke').}$ The lowest values for all three parameters were obtained in the integrated 'Bluecrop' and 'Nui'.

In strawberry fruits, organically grown 'Alba' and 'Clery' had the highest total phenolic contents (2.27 g GAE/kg and $2.04 \mathrm{~g} \mathrm{GAE} / \mathrm{kg}$, respectively), radical-scavenging activities (24.05 mmol TE/ $\mathrm{kg}$ and $23.35 \mathrm{mmol} \mathrm{TE} / \mathrm{kg}$, respectively), and total anthocyanin contents $(0.37 \mathrm{cy}-3-\mathrm{glu} / \mathrm{kg}$ and $0.33 \mathrm{cy}-3-\mathrm{glu} / \mathrm{kg}$, respectively). The radical-scavenging activities of strawberry leaves with a range from 802.29 to $2237.31 \mathrm{mmol} \mathrm{TE} / \mathrm{kg}$ were significantly higher when compared with the values for blueberry leaves (range: 336.19-679.94 mmol TE/kg) (Table 2). The TPC values ranged from 46.96 to $81.06 \mathrm{~g} \mathrm{GAE} / \mathrm{kg}$ in blueberry leaves, and the range was almost the same in strawberry leaves (38.22-82.25 g GAE/kg). All of the blueberry leaf samples from organic production had slightly higher TPC and RSA values when compared with integrated samples, while the distribution of these values was unequal between the integrated and organic strawberry leaves. 
Table 1. Contents of quantified phenolics (mg/kg) *, cis, trans-abscisic acid (mg/kg) *, Total Phenolic Content (TPC) (g GAE/kg) *, Radical-Scavenging Activity (RSA) $(\mathrm{mmol} \mathrm{TE} / \mathrm{kg}) *$, and Total Anthocyanin Content (TAC) $(\mathrm{g}$ cy-3-glu $/ \mathrm{kg}) *$ in fruits from three blueberry cultivars and three strawberry cultivars, West Serbia in 2014.

\begin{tabular}{|c|c|c|c|c|c|c|c|c|c|c|c|c|}
\hline \multirow{3}{*}{ Compound Name } & \multicolumn{6}{|c|}{ Blueberry Fruits } & \multicolumn{6}{|c|}{ Strawberry Fruits } \\
\hline & \multicolumn{3}{|c|}{ Integrated } & \multicolumn{3}{|c|}{ Organic } & \multicolumn{3}{|c|}{ Integrated } & \multicolumn{3}{|c|}{ Organic } \\
\hline & Bluecrop & Duke & Nui & Bluecrop & Duke & Nui & Alba & Favette & Clery & Alba & Favette & Clery \\
\hline Aesculin & 0.00 & 0.00 & 0.00 & $0.06^{b, * *}$ & $0.03^{c}$ & $0.11^{\mathrm{a}}$ & 0.00 & 0.00 & 0.00 & 0.00 & 0.00 & 0.00 \\
\hline 5-O-Caffeoylquinic Acid & $89.04^{c}$ & $33.08^{f}$ & $48.05^{\mathrm{d}}$ & $120.75^{a}$ & $33.73^{\mathrm{e}}$ & $119.84^{b}$ & 0.00 & 0.00 & 0.00 & 0.00 & 0.00 & 0.00 \\
\hline Epigallocatechin & $3.99^{\mathrm{a}}$ & $2.53^{d}$ & $3.00^{\mathrm{b}}$ & $2.92^{\mathrm{c}}$ & $2.10^{f}$ & $2.31^{\mathrm{e}}$ & 0.00 & 0.00 & 0.00 & 0.00 & 0.00 & 0.00 \\
\hline Catechin & $2.32^{\mathrm{e}}$ & $1.27^{\mathrm{i}}$ & $1.59 \mathrm{~g}$ & $1.43^{\mathrm{h}}$ & $0.67^{\mathrm{j}}$ & $1.45^{\mathrm{h}}$ & $3.19^{b}$ & $1.70^{\mathrm{f}}$ & $2.52^{\mathrm{c}}$ & $2.33^{e}$ & $2.43^{\mathrm{d}}$ & $3.79^{a}$ \\
\hline$p$-Hydroxybenzoic Acid & $0.54^{\mathrm{f}}$ & $0.80^{c}$ & $0.64^{\mathrm{e}}$ & $0.74^{\mathrm{d}}$ & $0.99^{\text {a }}$ & $1.00^{\mathrm{a}}$ & $0.32^{\mathrm{i}}$ & $0.96^{\mathrm{b}}$ & $0.51 \mathrm{~g}$ & $0.73^{\mathrm{d}}$ & $0.45^{\mathrm{h}}$ & $0.45^{\mathrm{h}}$ \\
\hline Caffeic Acid & $0.95^{\mathrm{c}}$ & $0.61^{\mathrm{d}}$ & $0.42^{f}$ & $2.39^{\mathrm{a}}$ & $1.26^{\mathrm{b}}$ & $2.36^{\mathrm{a}}$ & $0.33^{h}$ & $0.45^{\mathrm{e}}$ & $0.38^{\mathrm{g}}$ & $0.96^{\mathrm{c}}$ & $0.22^{\mathrm{i}}$ & $0.34^{\mathrm{h}}$ \\
\hline Epicatechin & $0.35^{\mathrm{a}}$ & $0.30^{\mathrm{b}}$ & $0.15^{\mathrm{e}}$ & $0.17^{\mathrm{d}}$ & $0.14^{\mathrm{f}}$ & $0.22^{c}$ & 0.00 & 0.00 & 0.00 & 0.00 & 0.00 & 0.00 \\
\hline Syringic Acid & $0.34^{\mathrm{d}}$ & $0.90^{\mathrm{b}}$ & $0.42^{c}$ & $0.25^{\mathrm{e}}$ & $1.27^{\mathrm{a}}$ & $0.43^{c}$ & 0.00 & 0.00 & 0.00 & 0.00 & 0.00 & 0.00 \\
\hline Apigenin 8-C-Glucoside & $0.73^{\mathrm{d}}$ & $0.57^{\mathrm{f}}$ & $0.75^{c}$ & $1.28^{\mathrm{b}}$ & $0.70^{\mathrm{e}}$ & $1.38^{\mathrm{a}}$ & 0.00 & 0.00 & 0.00 & 0.00 & 0.00 & 0.00 \\
\hline Quercetin 3-O-Rutinoside & $2.29^{d}$ & $0.95^{\mathrm{e}}$ & $3.31^{b}$ & $2.53^{c}$ & $0.81^{\mathrm{f}}$ & $4.65^{\mathrm{a}}$ & 0.00 & 0.00 & 0.00 & 0.00 & 0.00 & 0.00 \\
\hline$p$-Coumaric Acid & $0.14^{\mathrm{j}}$ & $0.13^{\mathrm{k}}$ & $0.08^{1}$ & $0.16^{\mathrm{i}}$ & $0.26^{\mathrm{h}}$ & $0.56^{\mathrm{g}}$ & $0.77^{\mathrm{e}}$ & $3.64^{b}$ & $1.05^{\mathrm{d}}$ & $5.27^{\mathrm{a}}$ & $0.59^{f}$ & $2.12^{\mathrm{c}}$ \\
\hline Quercetin 3-O-Galactoside & $30.92^{\mathrm{a}}$ & $25.39^{c}$ & $18.67^{\mathrm{f}}$ & $30.07^{\mathrm{b}}$ & $21.15^{\mathrm{d}}$ & $19.33^{\mathrm{e}}$ & $0.98^{i}$ & $0.38^{\mathrm{k}}$ & $0.21^{1}$ & $1.19^{\mathrm{h}}$ & $0.46^{j}$ & $1.26^{\mathrm{g}}$ \\
\hline Vanillic Acid & $0.13^{\mathrm{f}}$ & $0.27^{\mathrm{a}}$ & $0.11^{\mathrm{e}}$ & $0.15^{\mathrm{d}}$ & $0.23^{\mathrm{b}}$ & $0.21^{\mathrm{c}}$ & $0.05^{\mathrm{h}}$ & $0.06^{\mathrm{g}}$ & $0.04^{i}$ & $0.07^{g}$ & $0.05^{\mathrm{h}}$ & $0.02^{\mathrm{j}}$ \\
\hline Sinapic Acid & $0.11^{\mathrm{d}}$ & $0.14^{\mathrm{b}}$ & $0.05^{\mathrm{e}}$ & $0.13^{c}$ & $0.24^{\mathrm{a}}$ & $0.13^{c}$ & 0.00 & 0.00 & 0.00 & 0.00 & 0.00 & 0.00 \\
\hline Ferulic Acid & $0.28^{\mathrm{e}}$ & $0.35^{\mathrm{d}}$ & $0.18^{f}$ & $0.47^{\mathrm{c}}$ & $0.79^{b}$ & $1.70^{\mathrm{a}}$ & $0.01^{1}$ & $0.05^{\mathrm{i}}$ & $0.02^{k}$ & $0.17^{\mathrm{g}}$ & $0.08^{h}$ & $0.03^{j}$ \\
\hline Kaempferol 3-O-Glucoside & $0.84^{\mathrm{f}}$ & $0.11^{\mathrm{i}}$ & $0.16^{\mathrm{h}}$ & $0.83^{\mathrm{f}, \mathrm{g}}$ & $0.08^{j}$ & $0.17^{\mathrm{h}}$ & $2.17^{\mathrm{a}}$ & $1.03^{\mathrm{e}}$ & $0.82^{\mathrm{g}}$ & $1.54^{\mathrm{b}}$ & $1.34^{\mathrm{c}}$ & $1.17^{\mathrm{d}}$ \\
\hline Quercetin & $3.47^{g}$ & $10.12^{\mathrm{d}}$ & $2.88^{h}$ & $19.25^{c}$ & $53.69^{\text {a }}$ & $26.51^{b}$ & $3.54^{\mathrm{f}}$ & $2.46^{\mathrm{k}}$ & $2.45^{\mathrm{k}}$ & $3.99 \mathrm{e}$ & $2.57^{\mathrm{j}}$ & $2.77^{\mathrm{i}}$ \\
\hline Cinnamic Acid & $0.04^{\mathrm{h}}$ & $0.03^{\mathrm{i}}$ & $0.05^{\mathrm{g}}$ & $0.04^{\mathrm{h}}$ & $0.03^{\mathrm{i}}$ & $0.07^{\mathrm{f}}$ & $0.45^{\mathrm{c}}$ & $0.80^{\mathrm{b}}$ & $0.34^{\mathrm{d}}$ & $1.36^{\mathrm{a}}$ & $0.26^{\mathrm{e}}$ & $0.34^{\mathrm{d}}$ \\
\hline Naringenin & $0.28^{a}$ & $0.27^{\mathrm{b}}$ & $0.24^{\mathrm{d}}$ & $0.25^{c}$ & $0.28^{\mathrm{a}}$ & $0.25^{c}$ & 0.00 & 0.00 & 0.00 & 0.00 & 0.00 & 0.00 \\
\hline Kaempferol & 0.00 & 0.00 & 0.00 & $0.73^{\mathrm{d}}$ & $0.68^{f}$ & $0.61^{\mathrm{g}}$ & $0.80^{b}$ & $0.79^{b}$ & $0.77^{\mathrm{c}}$ & $1.74^{\mathrm{a}}$ & $0.70^{\mathrm{e}}$ & $0.71^{\mathrm{e}}$ \\
\hline cis, trans-Abscisic Acid & $1.40^{\mathrm{a}}$ & $0.70^{\mathrm{d}}$ & $0.97^{\mathrm{c}}$ & $1.04^{\mathrm{b}}$ & $0.25^{\mathrm{f}}$ & $0.65^{\mathrm{e}}$ & $0.04^{\mathrm{k}}$ & $0.13^{j}$ & $0.19^{\mathrm{h}}$ & $0.13^{j}$ & $0.24^{\mathrm{g}}$ & $0.16^{\mathrm{i}}$ \\
\hline TPC & $2.27^{f}$ & $4.08^{c}$ & $2.69^{\mathrm{e}}$ & $4.38^{\mathrm{b}}$ & $6.26^{\mathrm{a}}$ & $3.30^{\mathrm{d}}$ & $1.70^{\mathrm{h}}$ & $1.58^{\mathrm{i}}$ & $1.47^{\mathrm{j}}$ & $2.27^{\mathrm{f}}$ & $1.18^{\mathrm{k}}$ & $2.04 \mathrm{~g}$ \\
\hline RSA & $18.94 \mathrm{~g}$ & $25.36^{c}$ & $18.31^{\mathrm{h}}$ & $23.49^{\text {e }}$ & $33.83^{\text {a }}$ & $33.03^{\mathrm{b}}$ & $16.69^{\mathrm{k}}$ & $18.15^{\mathrm{i}}$ & $17.71^{\mathrm{j}}$ & $24.05^{\mathrm{d}}$ & $16.32^{1}$ & $23.35^{f}$ \\
\hline TAC & $0.62^{\mathrm{f}}$ & $1.80^{\mathrm{b}}$ & $0.82^{\mathrm{e}}$ & $1.05^{\mathrm{d}}$ & $2.86^{\mathrm{a}}$ & $1.63^{\mathrm{c}}$ & $0.16^{\mathrm{k}}$ & $0.23^{\mathrm{i}}$ & $0.22^{\mathrm{i}}$ & $0.37^{\mathrm{g}}$ & $0.19^{\mathrm{j}}$ & $0.33^{\mathrm{h}}$ \\
\hline
\end{tabular}

* The relative standard deviation (RSD) values were less than $5 \%$ for all the analysis. ${ }^{* *}$ Different letter in the same row denotes a significant difference among cultivars/cultivation systems according to Tukey's test, $p<0.05$. 
Table 2. Contents of quantified phenolics $(\mathrm{mg} / \mathrm{kg}) *$, TPC $(\mathrm{g} \mathrm{GAE} / \mathrm{kg}) *$ and RSA (mmol TE/kg) * in leaves from three blueberry cultivars and three strawberry cultivars, West Serbia in 2014.

\begin{tabular}{|c|c|c|c|c|c|c|c|c|c|c|c|c|}
\hline \multirow{3}{*}{ Compound Name } & \multicolumn{6}{|c|}{ Blueberry Leaves } & \multicolumn{6}{|c|}{ Strawberry Leaves } \\
\hline & \multicolumn{3}{|c|}{ Integrated } & \multicolumn{3}{|c|}{ Organic } & \multicolumn{3}{|c|}{ Integrated } & \multicolumn{3}{|c|}{ Organic } \\
\hline & Bluecrop & Duke & Nui & Bluecrop & Duke & Nui & Alba & Favette & Clery & Alba & Favette & Clery \\
\hline Gallocatechin & $21.29^{b, * *}$ & $15.63^{d}$ & $34.60^{a}$ & $17.79^{\mathrm{c}}$ & $14.11^{\mathrm{e}}$ & $11.17^{\mathrm{f}}$ & 0.00 & 0.00 & 0.00 & 0.00 & 0.00 & 0.00 \\
\hline Aesculin & $3.24^{c}$ & $2.57^{\mathrm{d}}$ & $2.54^{\mathrm{d}}$ & $3.41^{\mathrm{b}}$ & $2.40^{\mathrm{e}}$ & $3.70^{\mathrm{a}}$ & $0.94^{\mathrm{f}}$ & $0.64^{\mathrm{i}}$ & $0.75^{\mathrm{h}}$ & $0.83^{g}$ & $0.17^{\mathrm{k}}$ & $0.48^{j}$ \\
\hline 5-O-Caffeoylquinic Acid & $1826.07^{\mathrm{c}}$ & $1359.64^{\mathrm{f}}$ & $1617.33^{d}$ & $2108.73^{b}$ & $1485.93^{e}$ & $2380.63^{a}$ & $176.49^{g}$ & $176.64 \mathrm{~g}$ & $152.88^{\mathrm{h}}$ & $92.22^{\mathrm{i}}$ & $5.82^{\mathrm{k}}$ & $9.11^{\mathrm{j}}$ \\
\hline Catechin & $7.62 \mathrm{~g}$ & $6.00^{\mathrm{i}}$ & $13.50^{\mathrm{d}}$ & $7.57 \mathrm{~g}$ & $7.08^{h}$ & $4.51^{\mathrm{j}}$ & $0.31^{\mathrm{k}}$ & $9.47^{\mathrm{f}}$ & $11.67^{\mathrm{e}}$ & $14.69^{c}$ & $30.36^{b}$ & $31.86^{\mathrm{a}}$ \\
\hline$p$-Hydroxybenzoic Acid & $4.72^{\mathrm{d}}$ & $4.25^{\mathrm{e}}$ & $3.93^{f}$ & $5.86^{\mathrm{a}}$ & $4.80^{\mathrm{c}}$ & $4.90^{\mathrm{b}}$ & $1.68^{\mathrm{j}}$ & $1.96^{\mathrm{i}}$ & $1.52^{1}$ & $2.10^{\mathrm{h}}$ & $1.60^{\mathrm{k}}$ & $2.20 \mathrm{~g}$ \\
\hline Caffeic Acid & $55.51^{b}$ & $26.40^{f}$ & $41.76^{\mathrm{e}}$ & $51.16^{c}$ & $42.94^{\mathrm{d}}$ & $90.68^{a}$ & $5.21^{1}$ & $13.25 \mathrm{~g}$ & $6.76^{\mathrm{k}}$ & $8.11^{\mathrm{j}}$ & $12.23^{h}$ & $12.02^{\mathrm{i}}$ \\
\hline Syringic Acid & 0.00 & 0.00 & 0.00 & 0.00 & 0.00 & 0.00 & $0.48^{\mathrm{b}}$ & $0.30^{\mathrm{d}}$ & $0.30^{\mathrm{d}}$ & $0.24^{\mathrm{e}}$ & $0.63^{\mathrm{a}}$ & $0.33^{c}$ \\
\hline Apigenin 8-C-Glucoside & $10.06^{c}$ & $7.48^{\mathrm{f}}$ & $8.03^{\mathrm{e}}$ & $10.62^{b}$ & $9.68^{d}$ & $13.17^{\mathrm{a}}$ & 0.00 & 0.00 & 0.00 & 0.00 & 0.00 & 0.00 \\
\hline Quercetin 3-O-Rutinoside & $13.19^{d}$ & $6.63^{f}$ & $26.37^{b}$ & $12.73^{\mathrm{e}}$ & $14.22^{c}$ & $27.01^{\mathrm{a}}$ & 0.00 & 0.00 & 0.00 & 0.00 & 0.00 & 0.00 \\
\hline$p$-Coumaric Acid & $2.96^{\mathrm{i}}$ & $0.16^{\mathrm{k}}$ & $3.05^{h}$ & $3.76^{\mathrm{g}}$ & $2.40^{\mathrm{j}}$ & $3.81^{\mathrm{g}}$ & $5.15^{\mathrm{f}}$ & $10.36^{\mathrm{a}}$ & $6.09^{e}$ & $6.49^{\mathrm{d}}$ & $9.47^{\mathrm{b}}$ & $8.64^{c}$ \\
\hline Quercetin 3-O-Galactoside & $124.77^{\mathrm{b}}$ & $90.72^{f}$ & $99.65^{\mathrm{e}}$ & $125.67^{c}$ & $118.57^{\mathrm{d}}$ & $130.20^{\mathrm{a}}$ & $4.56^{\mathrm{k}}$ & $8.25^{\mathrm{j}}$ & $9.65^{h}$ & $9.36^{\mathrm{i}}$ & $12.53 \mathrm{~g}$ & $13.35^{\mathrm{g}}$ \\
\hline Vanillic Acid & $0.60^{\mathrm{h}}$ & $0.60^{\mathrm{h}}$ & $1.32^{\mathrm{d}}$ & $1.92^{\mathrm{b}}$ & $1.14^{\mathrm{f}}$ & $3.51^{\mathrm{a}}$ & $1.06^{\mathrm{g}}$ & $1.20^{\mathrm{e}}$ & $0.61^{\mathrm{h}}$ & $1.51^{\mathrm{c}}$ & $0.88^{\mathrm{i}}$ & $1.31^{\mathrm{d}}$ \\
\hline Sinapic Acid & $0.48^{\mathrm{g}}$ & $0.16^{\mathrm{i}}$ & $0.30^{\mathrm{h}}$ & $0.32^{\mathrm{h}}$ & $0.14^{\mathrm{j}}$ & $0.67^{\mathrm{f}}$ & $3.23^{\mathrm{e}}$ & $27.47^{\mathrm{a}}$ & $15.56^{\mathrm{b}}$ & $14.47^{c}$ & $0.04^{\mathrm{k}}$ & $5.64^{\mathrm{d}}$ \\
\hline Ferulic Acid & $0.99^{\mathrm{e}}$ & $0.48^{j}$ & $0.55^{\mathrm{i}}$ & $0.80^{\mathrm{g}}$ & $0.46^{\mathrm{k}}$ & $1.39^{\mathrm{c}}$ & $1.29^{\mathrm{d}}$ & $1.54^{\mathrm{b}}$ & $0.87^{\mathrm{f}}$ & $0.81^{\mathrm{g}}$ & $0.73^{\mathrm{h}}$ & $2.44^{\mathrm{a}}$ \\
\hline Kaempferol 3-O-Glucoside & $38.62^{b}$ & $4.09^{\mathrm{f}}$ & $11.10^{\mathrm{d}}$ & $41.07^{\mathrm{a}}$ & $6.77^{\mathrm{e}}$ & $12.65^{c}$ & $1.20^{\mathrm{k}}$ & $1.29^{\mathrm{j}}$ & $1.69^{\mathrm{i}}$ & $3.28 \mathrm{~g}$ & $2.41^{\mathrm{h}}$ & $0.52^{1}$ \\
\hline Quercetin & $151.49^{c}$ & $165.65^{b}$ & $89.51^{\mathrm{e}}$ & $83.57^{f}$ & $145.90^{\mathrm{d}}$ & $214.72^{\mathrm{a}}$ & $36.09 \mathrm{~g}$ & $20.82^{j}$ & $20.06^{k}$ & $21.67^{\mathrm{i}}$ & $32.35^{h}$ & $14.14^{1}$ \\
\hline Cinnamic Acid & $0.35^{\mathrm{g}}$ & $0.26^{\mathrm{i}}$ & $0.28^{h}$ & $0.09^{\mathrm{j}}$ & $0.27^{\mathrm{h}, \mathrm{i}}$ & $0.56^{\mathrm{e}, \mathrm{f}}$ & $0.57^{\mathrm{e}}$ & $1.04^{\mathrm{b}}$ & $0.55^{\mathrm{f}}$ & $0.66^{d}$ & $1.18^{\mathrm{a}}$ & $0.88^{c}$ \\
\hline Naringenin & $0.71^{\mathrm{e}}$ & $0.52^{j}$ & $0.66^{\mathrm{g}}$ & $0.65^{h}$ & $0.57^{\mathrm{i}}$ & $0.85^{b}$ & $0.68^{\mathrm{f}}$ & $0.83^{c}$ & $0.72^{\mathrm{e}}$ & $0.65^{\mathrm{h}}$ & $0.91^{\mathrm{a}}$ & $0.75^{\mathrm{d}}$ \\
\hline Kaempferol & $14.44^{\mathrm{a}}$ & $5.22^{\mathrm{d}}$ & $4.93^{\mathrm{e}}$ & $9.01^{b}$ & $4.00^{\mathrm{i}}$ & $8.25^{c}$ & $4.08^{g}$ & $2.83^{\mathrm{k}}$ & $3.13^{j}$ & $4.43^{\mathrm{f}}$ & $4.04^{\mathrm{h}}$ & $2.41^{1}$ \\
\hline Pinocembrin & 0.00 & 0.00 & 0.00 & 0.00 & 0.00 & 0.00 & $0.31^{b, c}$ & $0.33^{a}$ & $0.32^{b}$ & $0.30^{c, d}$ & $0.33^{a}$ & $0.29 \mathrm{~d}$ \\
\hline TPC & $54.56^{\mathrm{e}}$ & $46.96^{\mathrm{g}}$ & $55.75^{d}$ & $81.06^{\mathrm{b}}$ & $55.42^{d}$ & $77.65^{c}$ & $49.89^{\mathrm{f}}$ & $45.75^{\mathrm{h}}$ & $44.83^{i}$ & $38.22 \mathrm{k}$ & $41.29 \mathrm{j}$ & $82.25^{\mathrm{a}}$ \\
\hline RSA & $456.50^{\mathrm{i}}$ & $336.19^{1}$ & $423.25^{j}$ & $493.62^{\mathrm{h}}$ & $341.53^{k}$ & $679.94^{\mathrm{g}}$ & $802.29^{\mathrm{f}}$ & $1880.32^{b}$ & $1483.77^{\mathrm{c}}$ & $1225.43^{d}$ & $2237.31^{a}$ & $951.24{ }^{\mathrm{e}}$ \\
\hline
\end{tabular}

* The relative standard deviation (RSD) values were less than $5 \%$ for all the analysis. ** Different letter in the same row denotes a significant difference among cultivars/cultivation systems according to Tukey's test, $p<0.05$. 
In blueberry leaves, organically produced 'Bluecrop' had the highest TPC (81.06 g GAE/kg), followed by organic 'Nui' (77.65 g GAE/kg). Organically grown 'Nui' also stood out with significantly higher RSA ( $679.94 \mathrm{mmol} \mathrm{TE} / \mathrm{kg}$ ) when compared to the rest of the blueberry leaves. This is in line with the finding of Cezarotto et al. [48], who determined that TPC in 'Alice Blue', 'Flórida M', 'Bluegem', 'Clímax', and 'Powder blue' cultivars originated from Vaccinium ashei. Additionally, Wu et al. [49] determined the total phenolic content of 73 different blueberry leaves that included 'Bluecrop' (91.80 $\mathrm{g}$ GAE/kg), 'Nui' (114.6 g GAE/kg), and 'Duke' (78.71 g GAE/kg) cultivars that were in accordance with these results for organically grown samples, or higher. The results that were obtained herein for blueberries were lower when compared to six blueberry hybrids (derived from V. corymbosum, $V$. virgatum, and $V$. darrowii crosses) that were examined by Grace et al. [50]. In investigated hybrids, the results were in the range: $7.53-24.5 \mathrm{mg} \mathrm{GAE} / \mathrm{g}$.

The organically grown strawberry cultivar 'Clery' was characterized with the highest TPC $(82.25 \mathrm{~g}$ $\mathrm{GAE} / \mathrm{kg}$ ) in the leaves. TPC was almost uniform with the range from $38.22 \mathrm{~g} \mathrm{GAE} / \mathrm{kg}$ (organic 'Alba') to $49.89 \mathrm{~g} \mathrm{GAE} / \mathrm{kg}$ (in integrated 'Alba') in the rest of the analysed strawberry leaves, which coincides with the results of Buřičová et al. [51]. 'Favette' leaf samples from both growing regimes gave the highest RSA among all samples, with value of $2237.31 \mathrm{mmol} \mathrm{TE} / \mathrm{kg}$ in organic and $1880.32 \mathrm{mmol} \mathrm{TE} / \mathrm{kg}$ in the integrated regime.

\subsection{Determination of Phenolic Profile using UHPLC-LTQ Orbitrap MS ${ }^{4}$ Technique}

The investigated strawberry and blueberry extracts contained a wide range of phenolic compounds and the most abundant compounds were flavonol glycosides [16,52] and hydroxycinnamic and hydroxybenzoic acids in free, ester, and glycoside forms, as was expected [53,54]. In addition, a number of flavan-3-ols and procyanidins, as well as ellagic acid derivatives, were found in the extracts.

One of the goals of this study was obtaining insight into the metabolic profile of organic and integral strawberry and blueberry extracts while using the non-targeted approach, which resulted in the identification of total of 93 compounds (Table 3). The identification of compounds was based on the search for the $[\mathrm{M}-\mathrm{H}]^{-}$deprotonated molecule and its $\mathrm{MS}^{4}$ fragmentation. Table S1 shows the presence of identified compounds in organic and integral strawberry and blueberry fruit extracts, while, for the leaf extract, the presence is given in Table S2.

Hydroxycinnamic acid esters mainly quinic acid and various glycosides with characteristic fragmentation from the loss of sugar units (132 Da and 162 Da for pentosyl and hexosyl derivatives, respectively) were the most abundant compounds from the group of phenolic acids.

As was already mentioned, a large number of flavonols (kaempferol, quercetin, isorhamnetin, myricetin, and syringetin derivatives) were found in the tested fruit and leaf extracts. The determination of the glycosylation site in the case of flavonol 3-O or 7-O glycoside derivatives was achieved according to previously reported mass spectrometry rules $[55,56]$. Several flavonol glycosides have been identified as acyl derivatives with acetyl, malonyl, methyl-manonyl, and $p$-coumaroyl group linked to sugar moiety. Additionally, a number of hexuronyl derivatives have been identified with specific fragmentation (loss of $176 \mathrm{Da}$ ). For example, compound 24 at $639 \mathrm{~m} / \mathrm{z}$ and $5.45 \mathrm{~min}$. (Table 3) only identified in strawberry leaf samples was marked as quercetin 3-O-hexoside-7-O-hexuronide. It showed $\mathrm{MS}^{2}$ base peak at $463 \mathrm{~m} / \mathrm{z}$ formed by specific loss of hexuronyl group (176 Da) from the 7-O position. Further, MS ${ }^{3}$ base peak found at $301 \mathrm{~m} / \mathrm{z}$ (loss of hexosyl group-162 Da) and $\mathrm{MS}^{4}$ fragmentation confirmed the presence of quercetin as aglycone. At this point, it is interesting to mention compound 37 eluted at 6.14 min., which is structural isomer of compound 24 with same exact mass. It gave $\mathrm{MS}^{2}$ base peak at $301 \mathrm{~m} / \mathrm{z}$ (mass of deprotonated quercetin), generated by the elimination of hexosyl-hexuronide moiety and secondary MS ${ }^{2}$ peak at $337 \mathrm{~m} / z$, which corresponds to a mass of disaccharide residue without water. This compound, marked as quercetin 3-O-hexosyl-hexuronide, was only found in strawberry leaf samples. Figure S1 depicts the proposed structure and fragmentation pathway of compound 37. 
Table 3. Ultra-high performance liquid chromatography-MS ${ }^{4}$ (UHPLC-MS ${ }^{4}$ ) data about identification of main compounds in organic and integral strawberry and blueberry extracts.

\begin{tabular}{|c|c|c|c|c|c|c|c|c|c|}
\hline No & $t_{R}, \min$ & Compound Name & $\begin{array}{c}\text { Molecular Formula } \\
{[\mathbf{M}-\mathbf{H}]^{-}}\end{array}$ & $\begin{array}{l}\text { Calculated Mass } \\
{\left[\begin{array}{l}{[\mathbf{M}-\mathbf{H}]^{-}}\end{array}\right.}\end{array}$ & $\begin{array}{c}\text { Exact Mass } \\
{[\mathbf{M}-\mathbf{H}]^{-}}\end{array}$ & $\Delta \mathrm{ppm}$ & $\begin{array}{c}\text { MS }{ }^{2} \text { Fragments, } \\
\text { (\% Base Peak) }\end{array}$ & $\begin{array}{c}\text { MS }{ }^{3} \text { Fragments, } \\
\text { (\% Base Peak) }\end{array}$ & $\begin{array}{c}\text { MS }{ }^{4} \text { Fragments } \\
\text { (\% Base Peak) }\end{array}$ \\
\hline 1 & 2.94 & $\begin{array}{l}\text { Gallic Acid Hexoside } \\
\text { Isomer } 1\end{array}$ & $\mathrm{C}_{13} \mathrm{H}_{15} \mathrm{O}_{10^{-}}$ & 331.06707 & 331.06616 & 2.75 & $\begin{array}{c}294(10), \mathbf{1 6 9}(100), \\
125(5)\end{array}$ & 125(100) & 107(100), 81(10) \\
\hline 2 & 4.17 & $\begin{array}{c}\text { Dihydroxybenzoic } \\
\text { Acid hexoside Isomer } \\
1\end{array}$ & $\mathrm{C}_{13} \mathrm{H}_{15} \mathrm{O}_{9}^{-}$ & 315.07216 & 315.07104 & 3.55 & $\begin{array}{c}\text { 153(100), 152(50), } \\
\text { 109(15), 108(10) }\end{array}$ & 109(100) & $\begin{array}{l}\text { 123(25), 109(10), } \\
85(10), 81(100)\end{array}$ \\
\hline 3 & 4.31 & $\begin{array}{l}\text { Gallic Acid Hexoside } \\
\text { Isomer } 2\end{array}$ & $\mathrm{C}_{13} \mathrm{H}_{15} \mathrm{O}_{10}{ }^{-}$ & 331.06707 & 331.06622 & 2.57 & 169(100), 125(5) & 125(100) & $\begin{array}{l}\text { 110(10), 97(30), } \\
81(100), 53(30)\end{array}$ \\
\hline 4 & 4.44 & $\begin{array}{c}\text { Prodelphinidin Dimer } \\
\text { B Type }\end{array}$ & $\mathrm{C}_{30} \mathrm{H}_{25} \mathrm{O}_{13}{ }^{-}$ & 593.13006 & 593.12878 & 2.16 & $\begin{array}{c}467(15), 425(100), \\
407(30), 289(20)\end{array}$ & $\begin{array}{l}\text { 407(100), 381(5), } \\
273(10)\end{array}$ & $\begin{array}{l}389(30), 297(30), \\
285(100), 243(70)\end{array}$ \\
\hline 5 & 4.53 & Caffeoyltartaric Acid & $\mathrm{C}_{13} \mathrm{H}_{11} \mathrm{O}_{9}^{-}$ & 311.04031 & 311.03986 & 1.45 & $\begin{array}{c}\text { 179(50), 177(10) } \\
\mathbf{1 4 9}(100)\end{array}$ & $\begin{array}{c}131(60), 103(90), \\
87(100), 59(20)\end{array}$ & $59(100)$ \\
\hline 6 & 4.54 & $\begin{array}{l}\text { Chlorogenic Acid } \\
\text { Hexoside Isomer } 1\end{array}$ & $\mathrm{C}_{22} \mathrm{H}_{27} \mathrm{O}_{14}^{-}$ & 515.14008 & 515.14001 & 0.14 & $\begin{array}{c}353(100), 341(5), \\
323(10), 191(90) \\
179(5)\end{array}$ & 191(100), 179(10) & $\begin{array}{l}\text { 173(65), 127(80), } \\
111(30), 85(100)\end{array}$ \\
\hline 7 & 4.59 & Gallocatechin * & $\mathrm{C}_{15} \mathrm{H}_{13} \mathrm{O}_{7}^{-}$ & 305.06668 & 305.06537 & 4.29 & $\begin{array}{c}261(50), 221(70), \\
219(70), 179(100), \\
165(35)\end{array}$ & $\begin{array}{c}\text { 164(100), 151(40), } \\
135(30)\end{array}$ & $120(100), 108(20)$ \\
\hline 8 & 4.64 & $\begin{array}{c}\text { Dihydroxybenzoic } \\
\text { Acid } \\
\text { Hexosyl-Pentoside }\end{array}$ & $\mathrm{C}_{18} \mathrm{H}_{23} \mathrm{O}_{13}{ }^{-}$ & 447.11441 & 447.11353 & 1.97 & $\begin{array}{c}315(100), 285(10), \\
153(10)\end{array}$ & $153(100), 123(10)$ & $123(100)$ \\
\hline 9 & 4.71 & $\begin{array}{c}\text { Gallic Acid Hexoside } \\
\text { Isomer } 3\end{array}$ & $\mathrm{C}_{13} \mathrm{H}_{15} \mathrm{O}_{10^{-}}$ & 331.06707 & 331.06610 & 2.93 & $\begin{array}{l}313(100), 211(10), \\
169(30), 168(80), \\
150(10), 125(25)\end{array}$ & $\begin{array}{c}\text { 193(50), 151(100), } \\
125(80)\end{array}$ & $\begin{array}{c}123(100), 107(90) \\
95(65)\end{array}$ \\
\hline 10 & 4.72 & $\begin{array}{l}\text { Chlorogenic Acid } \\
\text { Hexoside Isomer } 2\end{array}$ & $\mathrm{C}_{22} \mathrm{H}_{27} \mathrm{O}_{14}{ }^{-}$ & 515.14008 & 515.13928 & 1.55 & $\begin{array}{c}353(40), 341(100), \\
335(30), 323(10), \\
191(15), 179(45)\end{array}$ & 179(100), 135(10) & 135(100) \\
\hline 11 & 4.83 & $\begin{array}{l}\text { Caffeic Acid Hexoside } \\
\text { Isomer } 1\end{array}$ & $\mathrm{C}_{15} \mathrm{H}_{17} \mathrm{O}_{9}^{-}$ & 341.08781 & 341.08685 & 2.81 & $\begin{array}{c}191(10), \mathbf{1 7 9}(100), \\
135(10)\end{array}$ & 135(100) & 135(100), 107(50) \\
\hline 12 & 4.84 & $\begin{array}{c}\text { Dihydroxybenzoic } \\
\text { Acid Pentoside }\end{array}$ & $\mathrm{C}_{12} \mathrm{H}_{13} \mathrm{O}_{8}^{-}$ & 285.06159 & 285.06094 & 2.28 & $\begin{array}{c}\text { 153(100), 152(25), } \\
109(5), 108(5)\end{array}$ & 109(100) & $81(100)$ \\
\hline 13 & 4.90 & $\begin{array}{l}\text { 3-O-Caffeoylquinic } \\
\text { Acid Isomer } 1\end{array}$ & $\mathrm{C}_{16} \mathrm{H}_{17} \mathrm{O}_{9}^{-}$ & 353.08781 & 353.08673 & 3.06 & $\begin{array}{c}\text { 191(100), 179(30), } \\
135(10)\end{array}$ & $\begin{array}{c}\text { 173(75), 127(100), } \\
111(40), 93(60) \\
85(90)\end{array}$ & $\begin{array}{c}109(30), 99(40) \\
85(100)\end{array}$ \\
\hline 14 & 5.02 & $\begin{array}{c}\text { Hydroxybenzoic Acid } \\
\text { Hexoside }\end{array}$ & $\mathrm{C}_{13} \mathrm{H}_{15} \mathrm{O}_{8}^{-}$ & 299.07724 & 299.07693 & 1.04 & 137(100) & $93(100)$ & - \\
\hline
\end{tabular}


Table 3. Cont

\begin{tabular}{|c|c|c|c|c|c|c|c|c|c|}
\hline No & $t_{\mathrm{R}}, \min$ & Compound Name & $\begin{array}{l}\text { Molecular Formula } \\
{[\mathrm{M}-\mathrm{H}]^{-}}\end{array}$ & $\begin{array}{l}\text { Calculated Mass } \\
{[\mathbf{M}-\mathbf{H}]^{-}}\end{array}$ & $\begin{array}{l}\text { Exact Mass } \\
{[\mathbf{M}-\mathbf{H}]^{-}}\end{array}$ & $\Delta \mathrm{ppm}$ & $\begin{array}{l}\text { MS }{ }^{2} \text { Fragments, } \\
\text { (\% Base Peak) }\end{array}$ & $\begin{array}{l}\text { MS }{ }^{3} \text { Fragments, } \\
\text { (\% Base Peak) }\end{array}$ & $\begin{array}{c}\text { MS }{ }^{4} \text { Fragments, } \\
\text { (\% Base Peak) }\end{array}$ \\
\hline 15 & 5.09 & $\begin{array}{l}\text { 3-O-Caffeoylquinic } \\
\text { Acid Isomer } 2\end{array}$ & $\mathrm{C}_{16} \mathrm{H}_{17} \mathrm{O}_{9}^{-}$ & 353.08781 & 353.08652 & 3.65 & $\begin{array}{c}\text { 191(100), 179(30), } \\
135(10)\end{array}$ & $\begin{array}{c}\text { 173(75), 127(100), } \\
111(40), 93(60) \\
85(90)\end{array}$ & $\begin{array}{c}109(30), 99(40) \\
85(100)\end{array}$ \\
\hline 16 & 5.10 & $\begin{array}{l}\text { Procyanidin Dimer B } \\
\text { Type Isomer } 1\end{array}$ & $\mathrm{C}_{30} \mathrm{H}_{25} \mathrm{O}_{12}^{-}$ & 577.13515 & 577.13434 & 1.40 & $\begin{array}{l}559(10), 451(30), \\
425(100), 407(40), \\
289(20), 287(10)\end{array}$ & $\begin{array}{l}\text { 407(100), 381(5), } \\
287(5), 273(10)\end{array}$ & $\begin{array}{l}389(30), 297(30) \\
285(100), 281(90)\end{array}$ \\
\hline 17 & 5.12 & Aesculin * & $\mathrm{C}_{15} \mathrm{H}_{15} \mathrm{O}_{9}^{-}$ & 339.07216 & 339.07114 & 3.01 & $\mathbf{1 7 7}(100)$ & $\begin{array}{c}\text { 177(5), 149(10), } \\
\text { 133(100), 105(10), } \\
89(5)\end{array}$ & $89(100)$ \\
\hline 18 & 5.28 & $\begin{array}{l}\text { Caffeic Acid Hexoside } \\
\text { Isomer } 2\end{array}$ & $\mathrm{C}_{15} \mathrm{H}_{17} \mathrm{O}_{9}^{-}$ & 341.08781 & 341.08664 & 3.43 & 179(100), 135(10) & 135(100) & 107(100), 79(20) \\
\hline 19 & 5.31 & $\begin{array}{l}\text { Coumaric Acid } \\
\text { Hexoside Isomer } 1\end{array}$ & $\mathrm{C}_{15} \mathrm{H}_{17} \mathrm{O}_{8}^{-}$ & 325.09289 & 325.09171 & 3.63 & 163(100), 119(10) & 119(100) & - \\
\hline 20 & 5.33 & $\begin{array}{l}\text { Procyanidin Dimer B } \\
\text { Type Isomer } 2\end{array}$ & $\mathrm{C}_{30} \mathrm{H}_{25} \mathrm{O}_{12}^{-}$ & 577.13515 & 577.13409 & 1.84 & $\begin{array}{c}559(5), 451(20), \\
\mathbf{4 2 5}(100), 407(35), \\
289(20), 287(10)\end{array}$ & $\begin{array}{l}\text { 407(100), 381(10), } \\
273(10)\end{array}$ & $\begin{array}{l}389(40), 297(40) \\
285(100), 243(75)\end{array}$ \\
\hline 21 & 5.35 & $\begin{array}{l}\text { 5-O-Caffeoylquinic } \\
\text { Acid * }\end{array}$ & $\mathrm{C}_{16} \mathrm{H}_{17} \mathrm{O}_{9}^{-}$ & 353.08781 & 353.08616 & 4.67 & 191(100), 179(5) & $\begin{array}{c}\text { 173(75), 127(100), } \\
111(40), 93(60) \\
85(90)\end{array}$ & $\begin{array}{c}109(40), 99(50) \\
85(100)\end{array}$ \\
\hline 22 & 5.37 & Epigallocatechin * & $\mathrm{C}_{15} \mathrm{H}_{13} \mathrm{O}_{7}^{-}$ & 305.06668 & 305.06589 & 2.59 & $\begin{array}{l}\text { 287(10), 261(40), } \\
247(20), 221(90), \\
219(80), \mathbf{1 7 9}(100)\end{array}$ & $\begin{array}{c}\text { 164(100), 151(40), } \\
135(30)\end{array}$ & $120(100), 108(20)$ \\
\hline 23 & 5.44 & $\begin{array}{c}\text { Dihydroxybenzoic } \\
\text { Acid Hexoside Isomer } \\
2\end{array}$ & $\mathrm{C}_{13} \mathrm{H}_{15} \mathrm{O}_{9}^{-}$ & 315.07216 & 315.07123 & 2.95 & $\begin{array}{c}\text { 153(100), 135(10), } \\
109(10)\end{array}$ & 135(100), 109(50) & 91(100) \\
\hline 24 & 5.45 & $\begin{array}{c}\text { Quercetin } \\
\text { 3-O-Hexoside-7-O- } \\
\text { hexuronide }\end{array}$ & $\mathrm{C}_{27} \mathrm{H}_{27} \mathrm{O}_{18}^{-}$ & 639.12029 & 639.11963 & 1.03 & $463(100), 301(20)$ & $343(5), \mathbf{3 0 1}(100)$ & $\begin{array}{c}179(70), 151(100), \\
107(10)\end{array}$ \\
\hline 25 & 5.47 & Catechin* & $\mathrm{C}_{15} \mathrm{H}_{13} \mathrm{O}_{6}{ }^{-}$ & 289.07176 & 289.07068 & 3.74 & $\begin{array}{c}271(5), 245(100) \\
205(40), 179(15) \\
125(5)\end{array}$ & $\begin{array}{c}227(30), 203(100), \\
187(25), 175(10) \\
161(20)\end{array}$ & $\begin{array}{c}188(70), 185(20), \\
175(100), 161(40), \\
157(10)\end{array}$ \\
\hline 26 & 5.48 & $\begin{array}{c}p \text {-Hydroxybenzoic } \\
\text { Acid * }\end{array}$ & $\mathrm{C}_{7} \mathrm{H}_{5} \mathrm{O}_{3}^{-}$ & 137.02442 & 137.02420 & 1.61 & 109(10), 93(100) & 93(10) & - \\
\hline 27 & 5.57 & $\begin{array}{c}\text { Coumaric Acid } \\
\text { Hexoside Isomer } 2\end{array}$ & $\mathrm{C}_{15} \mathrm{H}_{17} \mathrm{O}_{8}^{-}$ & 325.09289 & 325.09128 & 4.95 & $\begin{array}{l}289(20), 265(20), \\
235(10), 187(40), \\
163(80), \mathbf{1 4 5}(100)\end{array}$ & 117(100) & - \\
\hline 28 & 5.60 & $\begin{array}{c}\text { 4-O-Caffeoylquinic } \\
\text { Acid }\end{array}$ & $\mathrm{C}_{16} \mathrm{H}_{17} \mathrm{O}_{9}^{-}$ & 353.08781 & 353.08688 & 2.63 & $\begin{array}{l}\text { 191(60), 179(75), } \\
\text { 173(100), 135(15) }\end{array}$ & $\begin{array}{l}\text { 115(20), 111(50), } \\
93(100), 71(20)\end{array}$ & - \\
\hline
\end{tabular}


Table 3. Cont

\begin{tabular}{|c|c|c|c|c|c|c|c|c|c|}
\hline No & $t_{\mathrm{R}}, \min$ & Compound Name & $\begin{array}{l}\text { Molecular Formula } \\
{[\mathrm{M}-\mathrm{H}]^{-}}\end{array}$ & $\begin{array}{l}\text { Calculated Mass } \\
{[\mathbf{M}-\mathbf{H}]^{-}}\end{array}$ & $\begin{array}{l}\text { Exact Mass } \\
{[\mathbf{M}-\mathbf{H}]^{-}}\end{array}$ & $\Delta \mathrm{ppm}$ & $\begin{array}{l}\text { MS }{ }^{2} \text { Fragments, } \\
\text { (\% Base Peak) }\end{array}$ & $\begin{array}{c}\text { MS }{ }^{3} \text { Fragments, } \\
\text { (\% Base Peak) }\end{array}$ & $\begin{array}{c}\text { MS }{ }^{4} \text { Fragments, } \\
\text { (\% Base Peak) }\end{array}$ \\
\hline 29 & 5.61 & $\begin{array}{l}\text { Procyanidin Dimer B } \\
\text { Type Isomer } 3\end{array}$ & $\mathrm{C}_{30} \mathrm{H}_{25} \mathrm{O}_{12}^{-}$ & 577.13515 & 577.13312 & 3.52 & $\begin{array}{c}559(10), 451(20), \\
\text { 425(100), 407(40), } \\
289(20), 287(10)\end{array}$ & $\begin{array}{l}\text { 407(100), 381(5), } \\
\text { 273(10) }\end{array}$ & $\begin{array}{l}389(30), 297(30), \\
285(100), 243(75)\end{array}$ \\
\hline 30 & 5.80 & $\begin{array}{c}\text { Methyl } \\
\text { 3-caffeoylquinate }\end{array}$ & $\mathrm{C}_{17} \mathrm{H}_{19} \mathrm{O}_{9}{ }^{-}$ & 367.10346 & 367.10251 & 2.59 & $\begin{array}{c}\text { 193(20), 179(5), } \\
\text { 161(100), 135(10) }\end{array}$ & 133(100) & $77(100)$ \\
\hline 31 & 5.84 & Caffeic Acid * & $\mathrm{C}_{9} \mathrm{H}_{7} \mathrm{O}_{4}^{-}$ & 179.03498 & 179.03444 & 3.02 & 135(100) & $\begin{array}{c}\text { 135(60), 117(15), } \\
\text { 107(100), 91(55), } \\
79(15)\end{array}$ & - \\
\hline 32 & 5.92 & Epicatechin * & $\mathrm{C}_{15} \mathrm{H}_{13} \mathrm{O}_{6}^{-}$ & 289.07176 & 289.07104 & 2.49 & $\begin{array}{c}271(5), 245(100) \\
205(40), 179(15) \\
125(5)\end{array}$ & $\begin{array}{c}227(35), 203(100) \\
187(30), 175(15) \\
161(25)\end{array}$ & $\begin{array}{c}188(60), 185(20), \\
175(100), 161(35), \\
157(15)\end{array}$ \\
\hline 33 & 5.92 & $\begin{array}{l}\text { 5-Caffeoylquinic acid } \\
\text { Isomer }\end{array}$ & $\mathrm{C}_{16} \mathrm{H}_{17} \mathrm{O}_{9}^{-}$ & 353.08781 & 353.08624 & 4.45 & 191(100), 179(5) & $\begin{array}{c}\text { 173(75), 127(100), } \\
111(40), 93(60) \\
85(90)\end{array}$ & $\begin{array}{c}109(40), 99(50) \\
85(100)\end{array}$ \\
\hline 34 & 6.00 & Syringic Acid * & $\mathrm{C}_{9} \mathrm{H}_{9} \mathrm{O}_{5}^{-}$ & 197.04555 & 197.04477 & 3.96 & $\begin{array}{c}\text { 183(100), } 153(40) \\
138(10)\end{array}$ & $\begin{array}{c}\text { 167(100), 138(10), } \\
123(5)\end{array}$ & - \\
\hline 35 & 6.01 & Caffeoylshikimic Acid & $\mathrm{C}_{16} \mathrm{H}_{15} \mathrm{O}_{8}^{-}$ & 335.07724 & 335.07587 & 4.09 & $179(100), 135(25)$ & $135(100)$ & $107(100)$ \\
\hline 36 & 6.06 & $\begin{array}{c}\text { Myricetin } \\
\text { 3-O-rutinoside }\end{array}$ & $\mathrm{C}_{27} \mathrm{H}_{29} \mathrm{O}_{17^{-}}$ & 625.14102 & 625.14014 & 1.41 & $\begin{array}{c}607(10), 359(5) \\
329(5), 317(65) \\
316(100), 287(10)\end{array}$ & $\begin{array}{l}287(30), 271(100), \\
179(30), 151(10)\end{array}$ & $\begin{array}{l}271(10), 243(100) \text {, } \\
227(40), 215(15)\end{array}$ \\
\hline 37 & 6.14 & $\begin{array}{l}\text { Quercetin } \\
\text { 3-O-Hexosyl- } \\
\text { hexuronide }\end{array}$ & $\mathrm{C}_{27} \mathrm{H}_{27} \mathrm{O}_{18}^{-}$ & 639.12029 & 639.11865 & 2.57 & 337(10), 301(100) & $\begin{array}{l}\text { 273(20), 257(20), } \\
\text { 179(100), 151(75) }\end{array}$ & $151(100)$ \\
\hline 38 & 6.18 & $\begin{array}{c}\text { Myricetin } \\
\text { 3-O-hexoside }\end{array}$ & $\mathrm{C}_{21} \mathrm{H}_{19} \mathrm{O}_{13}{ }^{-}$ & 479.08311 & 479.08176 & 2.82 & $317(100), 316(80)$ & $\begin{array}{c}273(60), \mathbf{1 7 9}(100) \\
151(40)\end{array}$ & $151(100)$ \\
\hline 39 & 6.22 & $\begin{array}{c}\text { Methyl } \\
\text { 4-caffeoylquinate }\end{array}$ & $\mathrm{C}_{17} \mathrm{H}_{19} \mathrm{O}_{9}{ }^{-}$ & 367.10346 & 367.10211 & 3.68 & $\begin{array}{c}\text { 193(5), 179(5), } \\
\text { 161(100), 135(30) }\end{array}$ & 133(100) & 105(100) \\
\hline 40 & 6.26 & Ellagic Acid Pentoside & $\mathrm{C}_{19} \mathrm{H}_{13} \mathrm{O}_{12}^{-}$ & 433.04125 & 433.04047 & 1.80 & 301(100), 300(80) & $\begin{array}{c}301(95), 284(25) \\
257(100), 229(70), \\
222(15)\end{array}$ & $\begin{array}{l}229(70), 213(30), \\
201(15), 185(100)\end{array}$ \\
\hline 41 & 6.29 & $\begin{array}{c}\text { Methyl 3-p- } \\
\text { coumaroylquinate }\end{array}$ & $\mathrm{C}_{17} \mathrm{H}_{19} \mathrm{O}_{8}^{-}$ & 351.10854 & 351.10767 & 2.48 & $\begin{array}{l}\text { 163(5), 145(100), } \\
119(10), 117(10)\end{array}$ & $\mathbf{1 1 7}(100)$ & - \\
\hline 42 & 6.40 & $\begin{array}{l}\text { Ellagic Acid } \\
\text { Rhamnoside }\end{array}$ & $\mathrm{C}_{20} \mathrm{H}_{15} \mathrm{O}_{12}^{-}$ & 447.05690 & 447.05576 & 2.55 & 301(50), 300(100) & $\begin{array}{c}300(100), 284(15), \\
271(20), 257(30) \\
244(30)\end{array}$ & $\begin{array}{l}\text { 216(100), 200(40), } \\
188(15), 172(20)\end{array}$ \\
\hline 43 & 6.44 & $\begin{array}{l}\text { Apigenin } \\
\text { 8-C-glucoside * }\end{array}$ & $\mathrm{C}_{21} \mathrm{H}_{19} \mathrm{O}_{10}^{-}$ & 431.09837 & 431.09720 & 2.71 & $341(20), 311(100)$ & 283(100) & $\begin{array}{c}283(50), 239(100), \\
224(40), 197(50), \\
183(60)\end{array}$ \\
\hline
\end{tabular}


Table 3. Cont.

\begin{tabular}{|c|c|c|c|c|c|c|c|c|c|}
\hline No & $t_{\mathrm{R}}, \min$ & Compound Name & $\begin{array}{l}\text { Molecular Formula } \\
{[\mathrm{M}-\mathbf{H}]^{-}}\end{array}$ & $\begin{array}{l}\text { Calculated Mass } \\
{[\mathbf{M}-\mathbf{H}]^{-}}\end{array}$ & $\begin{array}{l}\text { Exact Mass } \\
{[\mathrm{M}-\mathbf{H}]^{-}}\end{array}$ & $\Delta \mathrm{ppm}$ & $\begin{array}{c}\text { MS }{ }^{2} \text { Fragments, } \\
(\% \text { Base Peak) }\end{array}$ & $\begin{array}{c}\text { MS }{ }^{3} \text { Fragments, } \\
\text { (\% Base Peak) }\end{array}$ & $\begin{array}{c}\text { MS }{ }^{4} \text { Fragments } \\
\text { (\% Base Peak) }\end{array}$ \\
\hline 44 & 6.45 & $\begin{array}{c}\text { Methyl } \\
\text { 5-caffeoylquinate } \\
\text { isomer } 1\end{array}$ & $\mathrm{C}_{17} \mathrm{H}_{19} \mathrm{O}_{9}^{-}$ & 367.10346 & 367.10190 & 4.25 & $\begin{array}{c}\text { 191(20), 179(100), } \\
161(10), 135(50)\end{array}$ & 135(100) & $\begin{array}{c}135(60), 107(100) \\
91(25), 79(20)\end{array}$ \\
\hline 45 & 6.46 & $\begin{array}{c}\text { Coumaric Acid } \\
\text { Hexoside Isomer } 3\end{array}$ & $\mathrm{C}_{15} \mathrm{H}_{17} \mathrm{O}_{8}^{-}$ & 325.09289 & 325.09180 & 3.35 & $\begin{array}{c}289(10), 265(10), \\
163(100), 161(50), \\
119(60), 101(20)\end{array}$ & $91(100)$ & - \\
\hline 46 & 6.47 & $\begin{array}{l}\text { Quercetin } \\
\text { 3-O-rutinoside * }\end{array}$ & $\mathrm{C}_{27} \mathrm{H}_{29} \mathrm{O}_{16}{ }^{-}$ & 609.14611 & 609.14496 & 1.89 & $\begin{array}{c}343(5), 301(100) \\
300(30), 271(10) \\
255(5)\end{array}$ & $\begin{array}{l}273(25), 257(20) \\
179(100), 151(75)\end{array}$ & $151(100)$ \\
\hline 47 & 6.50 & $\begin{array}{c}\text { Myricetin } \\
\text { 3-O-pentoside }\end{array}$ & $\mathrm{C}_{20} \mathrm{H}_{17} \mathrm{O}_{12}^{-}$ & 449.07255 & 449.07169 & 1.92 & $\begin{array}{c}\text { 387(5), 317(35) } \\
\text { 316(100) }\end{array}$ & $\begin{array}{l}\text { 287(30), 271(100), } \\
179(30), 151(10) \\
119(60), 101(20)\end{array}$ & $\begin{array}{r}271(10), 243(100) \\
227(40), 215(15)\end{array}$ \\
\hline 48 & 6.65 & $p$-Coumaric acid ${ }^{*}$ & $\mathrm{C}_{9} \mathrm{H}_{7} \mathrm{O}_{3}^{-}$ & 163.04007 & 163.03932 & 4.60 & 119(100) & $\begin{array}{c}93(25), 91(100) \\
72(10)\end{array}$ & - \\
\hline 49 & 6.67 & $\begin{array}{c}\text { Quercetin } \\
\text { 3-O-galactoside }\end{array}$ & $\mathrm{C}_{21} \mathrm{H}_{19} \mathrm{O}_{12}{ }^{-}$ & 463.08820 & 463.08719 & 2.18 & $301(100), 300(30)$ & $\begin{array}{l}273(25), 257(20) \\
179(100), 151(75)\end{array}$ & 151(100) \\
\hline 50 & 6.70 & $\begin{array}{l}\text { Methyl } \\
\text { 5-caffeoylquinate } \\
\text { Isomer } 2\end{array}$ & $\mathrm{C}_{17} \mathrm{H}_{19} \mathrm{O}_{9}^{-}$ & 367.10346 & 367.10269 & 2.10 & $\begin{array}{c}\text { 191(20), 179(100), } \\
161(10), 135(50)\end{array}$ & 135(100) & 109(100), 107(70) \\
\hline 51 & 6.75 & Ellagic Acid & $\mathrm{C}_{14} \mathrm{H}_{5} \mathrm{O}_{8}^{-}$ & 300.99899 & 300.99805 & 3.12 & $\begin{array}{c}284(40), 271(60), \\
257(100), 229(85), \\
185(40)\end{array}$ & $\begin{array}{c}\text { 229(100), 213(20) } \\
185(85)\end{array}$ & $\begin{array}{c}201(100), 185(95) \\
157(30), 145(20)\end{array}$ \\
\hline 52 & 6.82 & $\begin{array}{l}\text { Kaempferol } \\
\text { 7-O-rutinoside }\end{array}$ & $\mathrm{C}_{27} \mathrm{H}_{29} \mathrm{O}_{15}{ }^{-}$ & 593.15119 & 593.14972 & 2.48 & 285(100) & $\begin{array}{c}267(40), 257(100), \\
241(30), 229(40), \\
213(30)\end{array}$ & $\begin{array}{l}\text { 255(10), 239(30), } \\
229(100), 163(40)\end{array}$ \\
\hline 53 & 6.85 & $\begin{array}{l}\text { Quercetin } \\
\text { 3-O-rhamnosyl- } \\
\text { hexuronide }\end{array}$ & $\mathrm{C}_{27} \mathrm{H}_{27} \mathrm{O}_{17^{-}}$ & 623.12537 & 623.12341 & 3.15 & $\begin{array}{c}605(15), 491(10), \\
475(5), 315(40), \\
301(60), 300(100) \\
153(10), 152(80),\end{array}$ & $\begin{array}{l}\text { 271(100), 255(60), } \\
\text { 179(10), 151(10) }\end{array}$ & $\begin{array}{r}243(100), 227(80) \\
215(20), 199(20)\end{array}$ \\
\hline 54 & 6.87 & Vanillic Acid * & $\mathrm{C}_{8} \mathrm{H}_{7} \mathrm{O}_{4}^{-}$ & 167.03498 & 167.03419 & 4.73 & $\begin{array}{c}124(10), \mathbf{1 2 3}(100), \\
108(20)\end{array}$ & 108(100) & $79(100)$ \\
\hline 55 & 6.89 & $\begin{array}{l}\text { Isorhamnetin } \\
\text { 3-O-rutinoside }\end{array}$ & $\mathrm{C}_{28} \mathrm{H}_{31} \mathrm{O}_{16}{ }^{-}$ & 623.16176 & 623.16010 & 2.66 & $\begin{array}{c}315(100), 300(20), \\
271(10), 255(5)\end{array}$ & $\begin{array}{c}300(100), 287(5) \\
272(5)\end{array}$ & $\begin{array}{c}271(100), 255(50) \\
151(5)\end{array}$ \\
\hline 56 & 6.97 & $\begin{array}{c}\text { Quercetin } \\
\text { 3-O-pentoside }\end{array}$ & $\mathrm{C}_{20} \mathrm{H}_{17} \mathrm{O}_{11}{ }^{-}$ & 433.07763 & 433.07669 & 2.17 & $\begin{array}{c}\text { 343(5), 301(80) } \\
\mathbf{3 0 0 ( 1 0 0 )}\end{array}$ & $\begin{array}{c}\text { 271(100), 255(60), } \\
179(10), 151(10)\end{array}$ & $\begin{array}{r}243(100), 227(80) \\
215(20), 199(20)\end{array}$ \\
\hline 57 & 6.99 & Sinapic Acid * & $\mathrm{C}_{11} \mathrm{H}_{11} \mathrm{O}_{5}^{-}$ & 223.06120 & 223.06058 & 2.78 & $\begin{array}{c}\text { 208(100), 179(30), } \\
164(20)\end{array}$ & $\begin{array}{l}\text { 193(10), 164(100), } \\
149(15), 135(5)\end{array}$ & 149(100), 135(35) \\
\hline 58 & 7.02 & Ferulic Acid * & $\mathrm{C}_{10} \mathrm{H}_{9} \mathrm{O}_{4}^{-}$ & 193.05063 & 193.04990 & 3.78 & $\begin{array}{c}178(70), \mathbf{1 4 9}(100), \\
134(40)\end{array}$ & 134(100) & - \\
\hline
\end{tabular}


Table 3. Cont.

\begin{tabular}{|c|c|c|c|c|c|c|c|c|c|}
\hline No & $t_{\mathrm{R}}, \min$ & Compound Name & $\begin{array}{l}\text { Molecular Formula } \\
\qquad[\mathbf{M}-\mathbf{H}]^{-}\end{array}$ & $\begin{array}{l}\text { Calculated Mass } \\
{[\mathbf{M}-\mathbf{H}]^{-}}\end{array}$ & $\begin{array}{l}\text { Exact Mass } \\
{[\mathrm{M}-\mathbf{H}]^{-}}\end{array}$ & $\Delta \mathrm{ppm}$ & $\begin{array}{c}\text { MS }{ }^{2} \text { Fragments, } \\
(\% \text { Base Peak) }\end{array}$ & $\begin{array}{c}\text { MS }{ }^{3} \text { Fragments, } \\
\text { (\% Base Peak) }\end{array}$ & $\begin{array}{c}\text { MS }{ }^{4} \text { Fragments, } \\
\text { (\% Base Peak) }\end{array}$ \\
\hline 59 & 7.05 & $\begin{array}{c}\text { Methyl } \\
\text { 5-p-coumaroylquinate } \\
\text { Isomer 1 }\end{array}$ & $\mathrm{C}_{17} \mathrm{H}_{19} \mathrm{O}_{8}^{-}$ & 351.10854 & 351.10773 & 2.31 & $\begin{array}{c}\text { 163(100), } 145(5) \\
119(15)\end{array}$ & 119(100) & - \\
\hline 60 & 7.08 & $\begin{array}{c}\text { Kaempferol } \\
\text { 3-O-glucoside }\end{array}$ & $\mathrm{C}_{21} \mathrm{H}_{19} \mathrm{O}_{11}^{-}$ & 447.09329 & 447.09244 & 1.90 & $\begin{array}{l}327(20), 285(80), \\
284(100), 255(10)\end{array}$ & 255(100), 227(10) & $227(100), 211(60)$ \\
\hline 61 & 7.12 & $\begin{array}{c}\text { Syringetin } \\
\text { 3-O-hexoside }\end{array}$ & $\mathrm{C}_{23} \mathrm{H}_{23} \mathrm{O}_{13}^{-}$ & 507.11441 & 507.11292 & 2.94 & $\begin{array}{c}479(10), 387(20) \\
345(80), 344(100), \\
299(15)\end{array}$ & $\begin{array}{c}330(90), 316(100) \\
301(90), 287(10) \\
273(70)\end{array}$ & $\begin{array}{l}301(100), 300(20), \\
287(10), 273(60)\end{array}$ \\
\hline 62 & 7.16 & $\begin{array}{l}\text { Isorhamnetin } \\
\text { 3-O-hexoside }\end{array}$ & $\mathrm{C}_{22} \mathrm{H}_{21} \mathrm{O}_{12}^{-}$ & 477.10385 & 477.10321 & 1.34 & $\begin{array}{c}357(20), 315(50), \\
314(100), 300(5), \\
299(5), 285(10)\end{array}$ & $\begin{array}{c}300(30), 285(100), \\
271(75), 257(10) \\
243(25)\end{array}$ & $270(100)$ \\
\hline 63 & 7.20 & $\begin{array}{c}\text { Quercetin } \\
\text { 3-O-acetyl-hexoside } \\
\text { Isomer } 1\end{array}$ & $\mathrm{C}_{23} \mathrm{H}_{21} \mathrm{O}_{13}{ }^{-}$ & 505.09876 & 505.09756 & 2.38 & $\begin{array}{c}463(20), 343(20), \\
301(100), 300(60), \\
299(50)\end{array}$ & $\begin{array}{l}273(20), 257(20) \\
179(100), 151(75)\end{array}$ & 151(100) \\
\hline 64 & 7.24 & $\begin{array}{l}\text { Isorhamnetin } \\
\text { 3-O-hexuronide }\end{array}$ & $\mathrm{C}_{22} \mathrm{H}_{19} \mathrm{O}_{13}^{-}$ & 491.08311 & 491.08221 & 1.83 & $\begin{array}{l}473(10), 315(70) \\
301(100), 300(60)\end{array}$ & $\begin{array}{c}\text { 283(15), 272(20), } \\
256(10), \mathbf{1 7 9}(100), \\
151(75)\end{array}$ & 151(100) \\
\hline 65 & 7.29 & $\begin{array}{l}\text { Dicaffeoylquinic Acid } \\
\text { Isomer } 1\end{array}$ & $\mathrm{C}_{25} \mathrm{H}_{23} \mathrm{O}_{12}{ }^{-}$ & 515.11950 & 515.11789 & 3.13 & $353(100)$ & $\begin{array}{c}\text { 191(100), 179(40), } \\
135(10)\end{array}$ & $\begin{array}{c}173(100), 127(50), \\
111(40), 85(70)\end{array}$ \\
\hline 66 & 7.31 & $\begin{array}{c}\text { Methyl } \\
\text { 5-p-coumaroylquinate } \\
\text { Isomer 2 }\end{array}$ & $\mathrm{C}_{17} \mathrm{H}_{19} \mathrm{O}_{8}^{-}$ & 351.10854 & 351.10773 & 2.31 & $\begin{array}{c}\text { 163(100), 145(5), } \\
119(15)\end{array}$ & 119(100) & - \\
\hline 67 & 7.38 & $\begin{array}{c}\text { Quercetin } \\
\text { 3-O-acetyl-hexoside } \\
\text { Isomer } 2\end{array}$ & $\mathrm{C}_{23} \mathrm{H}_{21} \mathrm{O}_{13}{ }^{-}$ & 505.09876 & 505.09744 & 2.61 & $\begin{array}{l}463(30), 445(30), \\
343(5), 301(100), \\
300(90), 299(10)\end{array}$ & $\begin{array}{l}272(40), 256(25), \\
179(100), 151(75)\end{array}$ & 151(100) \\
\hline 68 & 7.44 & $\begin{array}{c}\text { Quercetin } \\
\text { 3-O-methyl-malonyl- } \\
\text { hexoside }\end{array}$ & $\mathrm{C}_{25} \mathrm{H}_{23} \mathrm{O}_{15}{ }^{-}$ & 563.10424 & 563.10309 & 2.04 & 531(100), 463(80) & $463(100)$ & $\begin{array}{c}343(5), 301(100) \\
300(30)\end{array}$ \\
\hline 69 & 7.45 & $\begin{array}{l}\text { Quercetin } \\
\text { 7-O-hexuronide }\end{array}$ & $\mathrm{C}_{21} \mathrm{H}_{17} \mathrm{O}_{13}{ }^{-}$ & 477.06692 & 477.06580 & 2.35 & 301(100) & $\begin{array}{c}273(20), 257(20) \\
179(100), 151(75)\end{array}$ & 151(100) \\
\hline 70 & 7.46 & $\begin{array}{l}\text { Isorhamnetin } \\
\text { 3-O-pentoside }\end{array}$ & $\mathrm{C}_{21} \mathrm{H}_{19} \mathrm{O}_{11}^{-}$ & 447.09329 & 447.09265 & 1.43 & $\begin{array}{c}357(10), 315(30), \\
314(100), 285(5), \\
271(5)\end{array}$ & $\begin{array}{c}300(10), 285(100), \\
271(70), 257(10) \\
243(20)\end{array}$ & $270(100)$ \\
\hline 71 & 7.49 & $\begin{array}{c}\text { Quercetin } \\
\text { 3-O-malonyl-hexoside }\end{array}$ & $\mathrm{C}_{24} \mathrm{H}_{21} \mathrm{O}_{15}{ }^{-}$ & 549.08859 & 549.08752 & 1.95 & $505(100)$ & $\begin{array}{c}463(30), 301(100) \\
300(50)\end{array}$ & $\begin{array}{l}273(15), 257(15), \\
179(100), 151(85)\end{array}$ \\
\hline 72 & 7.54 & $\begin{array}{l}\text { Dicaffeoylquinic Acid } \\
\text { Isomer } 2\end{array}$ & $\mathrm{C}_{25} \mathrm{H}_{23} \mathrm{O}_{12}^{-}$ & 515.11950 & 515.11865 & 1.65 & $353(100)$ & $\begin{array}{l}\text { 191(100), 179(40), } \\
173(20), 135(10)\end{array}$ & $\begin{array}{c}173(60), 127(100), \\
111(40), 85(80)\end{array}$ \\
\hline 73 & 7.60 & $\begin{array}{c}\text { Kaempferol } \\
\text { 7-O-hexuronide }\end{array}$ & $\mathrm{C}_{21} \mathrm{H}_{17} \mathrm{O}_{12}{ }^{-}$ & 461.07200 & 461.07141 & 1.28 & 285(100) & $\begin{array}{c}267(40), 257(100), \\
241(30), 229(50), \\
213(25)\end{array}$ & $\begin{array}{l}255(10), 239(30) \\
229(100), 163(60)\end{array}$ \\
\hline
\end{tabular}


Table 3. Cont.

\begin{tabular}{|c|c|c|c|c|c|c|c|c|c|}
\hline No & $t_{\mathrm{R}}, \min$ & Compound Name & $\begin{array}{l}\text { Molecular Formula } \\
\qquad[\mathrm{M}-\mathrm{H}]^{-}\end{array}$ & $\begin{array}{l}\text { Calculated Mass } \\
{[\mathbf{M}-\mathbf{H}]^{-}}\end{array}$ & $\begin{array}{l}\text { Exact Mass } \\
{[\mathrm{M}-\mathbf{H}]^{-}}\end{array}$ & $\Delta \mathrm{ppm}$ & $\begin{array}{l}\text { MS }{ }^{2} \text { Fragments, } \\
\text { (\% Base Peak) }\end{array}$ & $\begin{array}{l}\text { MS }{ }^{3} \text { Fragments, } \\
\text { (\% Base Peak) }\end{array}$ & $\begin{array}{c}\text { MS }{ }^{4} \text { Fragments, } \\
(\% \text { Base Peak) }\end{array}$ \\
\hline 74 & 7.63 & $\begin{array}{l}\text { Isorhamnetin 3-O- } \\
\text { malonyl-rutinoside }\end{array}$ & $\mathrm{C}_{31} \mathrm{H}_{33} \mathrm{O}_{19}{ }^{-}$ & 709.16160 & 709.16040 & 1.69 & 666(30), 665(100) & $\begin{array}{c}\text { 623(15), 315(100), } \\
300(20), 271(15), \\
255(10)\end{array}$ & $\begin{array}{l}300(100), 287(10), \\
272(10), 256(5)\end{array}$ \\
\hline 75 & 7.64 & Myricetin & $\mathrm{C}_{15} \mathrm{H}_{9} \mathrm{O}_{8}{ }^{-}$ & 317.03029 & 317.02927 & 3.22 & $\begin{array}{c}\text { 287(30), 271(15), } \\
193(10), \\
179(100), 1151(45)\end{array}$ & 151(100) & 107(100), 83(15) \\
\hline 76 & 7.70 & $\begin{array}{c}\text { Methyl } \\
\text { 3,4-dicaffeoylquinate }\end{array}$ & $\mathrm{C}_{26} \mathrm{H}_{25} \mathrm{O}_{12}^{-}$ & 529.13515 & 529.13397 & 2.23 & $367(100), 161(10)$ & $\begin{array}{c}\text { 335(5), 193(10), } \\
179(5), \mathbf{1 6 1}(100), \\
135(20)\end{array}$ & 133(100) \\
\hline 77 & 7.73 & $\begin{array}{l}\text { Kaempferol } \\
\text { 3-O-hexuronide } \\
\text { methyl Ether }\end{array}$ & $\mathrm{C}_{22} \mathrm{H}_{19} \mathrm{O}_{12}^{-}$ & 475.08820 & 475.08701 & 2.50 & $\begin{array}{c}327(10), 301(10), \\
285(70), 284(100), \\
255(35), 227(5)\end{array}$ & 255(100), 227(10) & $227(100), 211(60)$ \\
\hline 78 & 8.00 & $\begin{array}{c}\text { Kaempferol } \\
\text { 3-O-malonyl-hexoside }\end{array}$ & $\mathrm{C}_{24} \mathrm{H}_{21} \mathrm{O}_{14}{ }^{-}$ & 533.09368 & 533.09229 & 2.61 & 489(100) & $285(100)$ & $\begin{array}{l}267(40), 257(100), \\
241(30), 229(50)\end{array}$ \\
\hline 79 & 8.02 & Methyl Caffeate & $\mathrm{C}_{10} \mathrm{H}_{9} \mathrm{O}_{4}^{-}$ & 193.05063 & 193.05019 & 2.28 & $\begin{array}{l}\text { 178(30), 161(100), } \\
134(70), 111(10)\end{array}$ & 133(100) & - \\
\hline 80 & 8.04 & $\begin{array}{c}\text { Methyl } \\
\text { 3,5-dicaffeoylquinate }\end{array}$ & $\mathrm{C}_{26} \mathrm{H}_{25} \mathrm{O}_{12}{ }^{-}$ & 529.13515 & 529.13391 & 2.34 & $\begin{array}{c}367(100), 349(10), \\
179(10), 161(10)\end{array}$ & $\begin{array}{c}\text { 335(5), 193(10), } \\
191(25), \mathbf{1 7 9}(100) \\
161(80)\end{array}$ & $135(100)$ \\
\hline 81 & 8.16 & $\begin{array}{l}\text { Feruloyl-coumaroylquinic } \\
\text { acid isomer } 1\end{array}$ & $\mathrm{C}_{26} \mathrm{H}_{25} \mathrm{O}_{11}^{-}$ & 513.14024 & 513.13953 & 1.38 & $\begin{array}{c}367(100), 351(5) \\
161(10)\end{array}$ & $\begin{array}{c}335(5), 193(10) \\
179(5), \mathbf{1 6 1}(100) \\
135(30)\end{array}$ & 133(100) \\
\hline 82 & 8.29 & $\begin{array}{c}\text { Methyl } \\
\text { 4,5-dicaffeoylquinate }\end{array}$ & $\mathrm{C}_{26} \mathrm{H}_{25} \mathrm{O}_{12}^{-}$ & 529.13515 & 529.13385 & 2.46 & $\begin{array}{c}367(100), 349(10), \\
179(15), 161(10)\end{array}$ & $\begin{array}{c}335(10), 193(10), \\
191(20), \mathbf{1 7 9}(100), \\
161(80)\end{array}$ & $135(100)$ \\
\hline 83 & 8.30 & $\begin{array}{l}\text { Kaempferol3-O-p- } \\
\text { coumaroyl-hexoside }\end{array}$ & $\mathrm{C}_{30} \mathrm{H}_{25} \mathrm{O}_{13}{ }^{-}$ & 593.13006 & 593.12769 & 4.00 & $\begin{array}{l}447(15), 307(10) \\
285(100)\end{array}$ & $\begin{array}{c}\text { 257(100), 241(50), } \\
229(35), 213(40), \\
151(90)\end{array}$ & $\begin{array}{l}256(10), 239(25), \\
229(100), 213(20)\end{array}$ \\
\hline 84 & 8.49 & cis, trans-Abscisic acid & $\mathrm{C}_{15} \mathrm{H}_{19} \mathrm{O}_{4}^{-}$ & 263.12888 & 263.12839 & 1.86 & $\begin{array}{c}\text { 219(15), 153(100), } \\
151(5)\end{array}$ & $\begin{array}{c}\text { 138(100), 109(10), } \\
97(15)\end{array}$ & $122(100)$ \\
\hline 85 & 8.53 & $\begin{array}{l}\text { Feruloyl-coumaroylquinic } \\
\text { Acid Isomer } 2\end{array}$ & $\mathrm{C}_{26} \mathrm{H}_{25} \mathrm{O}_{11}^{-}$ & 513.14024 & 513.13904 & 2.34 & $\begin{array}{c}367(70), 349(100), \\
179(5), 161(10)\end{array}$ & $\begin{array}{l}\text { 305(20), 179(10), } \\
\mathbf{1 6 1}(100), 133(15)\end{array}$ & 133(100) \\
\hline 86 & 8.62 & Quercetin* & $\mathrm{C}_{15} \mathrm{H}_{9} \mathrm{O}_{7}^{-}$ & 301.03538 & 301.03442 & 3.19 & $\begin{array}{c}271(50), 255(20), \\
179(100), 151(80), \\
107(5)\end{array}$ & 151(100) & 107(100), 83(10) \\
\hline 87 & 8.78 & $\begin{array}{l}\text { Feruloyl-coumaroylquinic } \\
\text { Acid Isomer } 3\end{array}$ & $\mathrm{C}_{26} \mathrm{H}_{25} \mathrm{O}_{11}^{-}$ & 513.14024 & 513.14008 & 0.31 & $\begin{array}{c}367(70), 349(100), \\
337(10), 179(5), \\
163(15), 161(10)\end{array}$ & $\begin{array}{c}\text { 305(10), 193(20), } \\
173(15), \mathbf{1 6 1}(100), \\
133(10)\end{array}$ & $133(100)$ \\
\hline 88 & 9.03 & Cinnamic Acid * & $\mathrm{C}_{9} \mathrm{H}_{7} \mathrm{O}_{2}^{-}$ & 147.04515 & 147.04463 & 3.54 & $\begin{array}{c}\text { 104(10), 103(100), } \\
87(10)\end{array}$ & 119(100) & - \\
\hline
\end{tabular}


Table 3. Cont.

\begin{tabular}{|c|c|c|c|c|c|c|c|c|c|}
\hline No & $t_{R}, \min$ & Compound Name & $\begin{array}{l}\text { Molecular Formula } \\
\qquad[\mathrm{M}-\mathrm{H}]^{-}\end{array}$ & $\begin{array}{l}\text { Calculated Mass } \\
\qquad[\mathbf{M}-\mathbf{H}]^{-}\end{array}$ & $\begin{array}{l}\text { Exact Mass } \\
{[\mathbf{M}-\mathbf{H}]^{-}}\end{array}$ & $\Delta \mathrm{ppm}$ & $\begin{array}{l}\text { MS }{ }^{2} \text { Fragments, } \\
\text { (\% Base Peak) }\end{array}$ & $\begin{array}{l}\text { MS }{ }^{3} \text { Fragments, } \\
\text { (\% Base Peak) }\end{array}$ & $\begin{array}{c}\text { MS }{ }^{4} \text { Fragments, } \\
\text { (\% Base Peak) }\end{array}$ \\
\hline 89 & 9.32 & Naringenin * & $\mathrm{C}_{15} \mathrm{H}_{11} \mathrm{O}_{5}^{-}$ & 271.06120 & 271.06039 & 2.99 & 177(10), 151(100) & \multirow{2}{*}{$\begin{array}{c}107(100) \\
\mathbf{2 1 1}(100), 195(5) \\
167(15)\end{array}$} & $65(100)$ \\
\hline 90 & 9.51 & Kaempferol * & $\mathrm{C}_{15} \mathrm{H}_{9} \mathrm{O}_{6}^{-}$ & 285.04046 & 285.03909 & 4.81 & $255(100), 227(10)$ & & $211(40), 137(100)$ \\
\hline 91 & 9.57 & Syringetin & $\mathrm{C}_{17} \mathrm{H}_{13} \mathrm{O}_{8}^{-}$ & 345.06159 & 345.06036 & 3.56 & $\begin{array}{c}330(100), 315(10) \\
300(5)\end{array}$ & $315(100)$ & $\begin{array}{l}\text { 287(100), 271(40), } \\
259(25), 243(15)\end{array}$ \\
\hline 92 & 9.69 & Isorhamnetin & $\mathrm{C}_{16} \mathrm{H}_{11} \mathrm{O}_{7}^{-}$ & 315.05103 & 315.04985 & 3.75 & $301(20), 300(100)$ & $\begin{array}{l}\text { 283(40), 271(80) } \\
\text { 255(30), 227(30), } \\
\text { 151(100) }\end{array}$ & 107(100), 83(15) \\
\hline 93 & 11.46 & Pinocembrin * & $\mathrm{C}_{15} \mathrm{H}_{11} \mathrm{O}_{4}^{-}$ & 255.06628 & 255.06580 & 1.88 & $\begin{array}{c}\text { 213(100), 187(15), } \\
151(30), 145(10) \\
107(5)\end{array}$ & $\begin{array}{c}185(100), 169(20) \\
145(20)\end{array}$ & $\begin{array}{l}\text { 185(10), 157(15), } \\
143(100), 141(50)\end{array}$ \\
\hline
\end{tabular}

* Conformed using available standards; The other compounds were identified using HRMS and MS ${ }^{\mathrm{n}}$ data available in literature; $t_{\mathrm{R}}$-retention time; $\Delta$ ppm—Mean mass accuracy. " $-{ }^{\prime \prime}$ Stands for not detected fragment. 


\subsection{Differences in Strawberry and Blueberry Phenolic Profilesfrom Fruits and Leaves}

A total of 20 phenols were quantified in blueberry and 11 in strawberry fruit samples, according to the results that are presented in Table 1 . Aesculin, 5-O-caffeoylquinic acid, epigallocatechin, epicatechin, syringic acid, apigenin 8-C-glucoside, quercetin 3-O-rutinoside, sinapic acid, and naringenin were found only in blueberries, and they could be potentially used as a marker for blueberry products. As for phenols that were identified in all fruit samples, the contents of the majority of them were higher in blueberries than in strawberries, with the exception of catechin, $p$-coumaric acid, kaempferol 3-O-glucoside, and cinnamic acid. 5-O-caffeoylquinic acid was the most abundant phenolic compound in blueberries, followed by quercetin 3-O-galactoside, with the average contents of $74.08 \mathrm{mg} / \mathrm{kg}$ and $24.25 \mathrm{mg} / \mathrm{kg}$, respectively. Caffeoylquinic acids and quercetin 3-O-galactosideare were both synthesized in plants as a response to oxidative stresses, and they are beneficial for human health limiting atherosclerosis and carcinogenesis and anti-inflammatory activities [57,58]. Contrary to our study, Zimmer et al. [59], reported that the most abundant phenolic compound in blueberry cultivars 'Briteblue', 'Bluegem' and 'Woodard' was chlorogenic acid and (was not detected in this study). Another study that was conducted in China [60] proved that ferulic acid was the most dominant compound among the phenols in blueberries. The reason for such diverse results is, most probably, genetic predisposition, and/or agroecological conditions where the study was undertaken. However, Häkkinen and Törrönen [42] and Howard et al. [61] concluded that genetics play a more significant role in the synthesis of the phenolic compounds than growing conditions. Routray and Orsat [21] suggested that abiotic factors and changes in environmental conditions during the season could explain results variations. In the case of strawberries, quantification revealed catechin, quercetin, and kaempferol 3-O-glucoside as the dominant compounds. Additionally, Wang and Millner [62] showed that quercetin, and kaempferol, are some of the main phenolic compounds found in strawberry fruit. Generally, both components are dominant flavonoids in fruits and effective antioxidants, playing a protective role against cardiovascular diseases and anti-inflammatory and anti-carcinogenic activities [63].

The characterization of phenols in leaves (Table 2) revealed a similar amount of phenolic compounds in blueberry (18 in total) and strawberries (17 in total). 5-O-caffeoylquinic acid, quercetin 3-O-galactoside, caffeic acid, and quercetin were the most abundant, while gallocatechin, apigenin 8-C-glucoside, and quercetin 3-O-rutinoside were exclusively found in the blueberry leaf samples. Skupień et al. [64] stated that the main substances present in blueberry (V. corymbosum L.) leaf extracts were caffeic acid, querectin, kaempferol, and gallic acid, while Harris et al. [65], for V. angustifolium, showed chlorogenic acid, epicatechin, catechin, caffeic acid, and quercetin as the main constituents of leaf extracts. According to Oszmianskiet al. [66], the most abundant phenols in leaves of native blueberry in Europe were chlorogenic acid and quercetin 3-O-glucuronide. Li et al. [67] found that caffeoylquinic acid and quercetin were the most predominant phenol groups in the blueberry leaves in China. In addition, chlorogenic acid and rutin were the major phenolic compounds in the leaves of blueberry that were cultivated in Japan. This could be clarified by statements that were given by Kaur et al. [68] and Cheel et al. [69], where the composition of secondary metabolites in a plant depend on various factors, e.g., genetics, climatic conditions, growing site, harvesting time, and others.

Besides the high level of 5-O-caffeoylquinic acid, quercetin and catechin, strawberry leaves were unique by the presence of some quantity of syringic acid and pinocembrin. These data are in accordance with the results of Buřičová et al. [51], where catechin was detected as the dominant compound that was present in the extracts of strawberry leaves. The contents of seven phenols (5-O-caffeoylquinic acid, caffeic acid, quercetin 3-O-galactoside, aesculin, $p$-hydroxybenzoic acid, kaempferol 3-O-glucoside, and quercetin) were significantly higher in all of the investigated blueberry leaves than in strawberry samples, while the opposite trend was observed in the case of $p$-coumaric acid. 


\subsection{Phenolic Profiles of Plants from Organic and Integrated Production}

\subsubsection{Blueberry Fruits}

Certain differences were found when the organic and integrated grown regimes were compared (Table 1). Blueberries from organic production stored higher levels of quercetin, apigenin 8-C-glucoside, and six phenolic acids, namely, 5-O-caffeoylquinic, $p$-hydroxybenzoic, caffeic, $p$-coumaric, sinapic, and ferulic acid. Aesculin and kaempferol were only detected in organically grown blueberries, with the average contents of $0.07 \mathrm{mg} / \mathrm{kg}$ and $0.67 \mathrm{mg} / \mathrm{kg}$, respectively. On the contrary, the integrated fruit samples had higher amounts of two flavan-3-ols (catechin and epigallocatechin).

Cinnamic acid was detected in almost equal amounts in all blueberries, regardless of the production regimes (range from $0.03 \mathrm{mg} / \mathrm{kg}$ to $0.07 \mathrm{mg} / \mathrm{kg}$ ), and the same trend was in naringenin $(0.24-0.28 \mathrm{mg} / \mathrm{kg}$ ). 'Nui' blueberry from organic production was distinguished from the integrated one by higher contents of the most of the phenols. The exceptions were catechin and epigallocatechin, whose contents were higher in integrated 'Nui'.

\subsubsection{Strawberry Fruits}

All three organically grown strawberry cultivars stored higher amounts of ferulic acid, quercetin, and quercetin 3-O-galactoside (Table 1). On the other hand, there was no phenol whose content was higher in all the integrated strawberry fruits. 'Alba' was the only cultivar that was characterized with higher contents of almost all quantified phenolic compounds in organically produced fruit. Moreover, the extract of 'Alba' stood out according to the highest contents of several phenolic acids (caffeic acid, $p$-coumaric acid, vanillic acid, ferulic acid, and cinnamic acid), and two flavonols (kaempferol and quercetin). Higher contents of some phenolic acids, such as $p$-hydroxybenzoic acid, caffeic acid, $p$-coumaric acid, and cinnamic acid, were found in 'Favette' from integrated regime in comparison to organic.

\subsubsection{Blueberry Leaves}

The most abundant phenolic compound was 5-O-caffeoylquinic acid, with the range from 1359.64 to $2380.63 \mathrm{mg} / \mathrm{kg}$, followed by quercetin 3-O-galactoside and quercetin in all blueberry leaves (Table 2) (ranges: $90.72-130.20 \mathrm{mg} / \mathrm{kg}$ and $83.57-214.72 \mathrm{mg} / \mathrm{kg}$, respectively). The levels of the two major phenols, 5-O-caffeoylquinic acid and quercetin 3-O-galactoside, were higher in all of the blueberry leaves from organic production, in comparison with the integrated regime. The same tendency was observed with three phenolic acids ( $p$-hydroxybenzoic, $p$-coumaric, and vanillic acid), apigenin 8 - $C$-glucoside, and kaempferol 3-O-glucoside. On the other hand, quercetin, which was another major phenol, was found in higher amounts in 'Bluecrop' and 'Duke' leaves from integrated production, and organically grown 'Nui'. By further comparison, it was observed that gallocatechin was the only phenol that was quantified in higher contents in all leaf samples from integrated production.

Organically grown 'Nui' stored higher amounts of all phenols, with the exception of gallocatechin and catechin, when compared to the 'Nui' leaves from integrated production. Moreover, it should be pointed out that organic 'Nui' stood out with the highest amounts of the majority of phenols in comparison to all the other blueberry leaf samples. Organically grown 'Duke' was also characterised with higher contents of the most of the phenolic compound (twelve) when compared to the integrated 'Duke' samples. The opposite trend was observed with 'Bluecrop' samples, as ten of eighteen phenolics were present in higher amounts in the integrated 'Bluecrop' leaves.

\subsubsection{Strawberry Leaves}

The amounts of catechin and cinnamic acid were higher in the organic leaves when compared to integrated (Table 2). On the contrary, all of the integrated samples stored higher amounts of 5-O-caffeoylquinic acid and aesculin in comparison to the organically grown ones, while the content of pinocembrin was fairly uniform in the samples from both regimes (range from 0.29 to $0.33 \mathrm{mg} / \mathrm{kg}$ ). 
5-O-caffeoylquinic acid stood out as the most abundant phenolic compound in strawberry leaves from integrated production, with the average concentration amounting to $168.67 \mathrm{mg} / \mathrm{kg}$. This phenolic acid was also the dominant compound in organic 'Alba' $(92.22 \mathrm{mg} / \mathrm{kg})$. In organically grown 'Clery', the prevalent was catechin $(31.86 \mathrm{mg} / \mathrm{kg})$, while, in organic 'Favette', these were catechin $(30.36 \mathrm{mg} / \mathrm{kg})$ and quercetin $(32.35 \mathrm{mg} / \mathrm{kg})$. Interestingly, the highest and lowest contents of sinapic acid were observed in 'Favette' samples, concentration in integrated leaves amounted $27.47 \mathrm{mg} / \mathrm{kg}$, while it was $0.04 \mathrm{mg} / \mathrm{kg}$ in organic.

\subsection{Cis, trans-Abscisic Acid}

cis, trans-Abscisic acid (ABA) is a hormone that is ubiquitous in plants, but it can be also found from sponges to humans [70]. In plant metabolism, it regulates plant growth and development, cell wall metabolism, responses to biotic and abiotic stresses (pests, cold, drought, salinity, UV radiation), change in gene expression, and adaptive physiological responses, bud and seed dormancy, seed and pollen germination, fruit ripening and senescence, root geotropism, stomatal functions, quality formation, sugar and acid metabolism, and phenolic metabolism [71-73]. ABA is a ripening promoter that influences fruit softening, aroma, anthocyanin biosynthesis, and growth in both climacteric (blueberry) and non-climacteric fruits (strawberry) [74].

In our study, ABA was detected in all fruit samples (Table 1), while its presence did not characterize the leaves. Higher amounts were observed in blueberries (climacteric fruit, which continues to ripe after harvesting) when compared with strawberries (non-climacteric fruit) and the average values were 0.84 and $0.15 \mathrm{mg} / \mathrm{kg}$, respectively. These results were expected, as it was documented that the content of ABA in climacteric fruits increases from maturation to harvest, while it increases before maturation and decreases until harvest in non-climacteric fruits [75]. When results that were obtained for blueberries from the two growing regimes were compared, significantly higher contents of ABA were found in integrated fruits. The highest contents were detected in the 'Bluecrop' samples, both integrated $(1.40 \mathrm{mg} / \mathrm{kg})$ and organic $(1.04 \mathrm{mg} / \mathrm{kg})$, and the lowest in cultivar 'Duke' (integrated: $0.70 \mathrm{mg} / \mathrm{kg}$; organic: $0.25 \mathrm{mg} / \mathrm{kg}$ ). Regarding the fact that ABA is a stress hormone [76] that is synthesized in response to many kinds of stresses, it can be hypothesized that the synthesis of this hormone is cultivar dependent, concluding that 'Bluecrop' could be a more stress sensitive cultivar. In strawberry fruits, organically grown 'Alba' and 'Favette' were richer in ABA when compared with integrated production.

According to Harris [77], ABA is controlling different aspects of root architecture, such as cell division and cell elongation. The root system of strawberry is very complex, having primary roots (that are mainly used for storage), secondary roots (used for water and nutrient movement), and thousands of root-hairs, which are important for water and nutrients capturing from the soil. Blueberries have a shallow and fibrous root system without any root-hairs. The synthesis of ABA is influenced by different root architecture and physiology of root growth in those two fruit species.

\subsection{Leaf (Source)-Fruit (Sink) Relationship}

The chemical analysis of leaves and fruits from organic and integrated production showed that fruits from both fruit species had much lower TPC and RSA when compared to leaves. 'Blueberry' leaves had, on average, 19 fold higher TPC when compared to fruits, while, in strawberry, it was 30 fold higher. Similar results were obtained for RSA, where in blueberry RSA was, on average, 19 folds higher in leaves and, in strawberry, 77 folds higher in leaves when compared to fruits. Ehlenfeldt and Prior proved similar results for blueberries [78], where leaves of Vaccinium corymbosum cultivars had much higher phenolics values then fruits. All of the individual phenolic compounds that were present in both organs were much higher in leaves in contrast to fruits. The biggest differences were determined for caffeic acid, (up to 100 folds higher in leaves of integrated 'Nui' as compared to fruits), quercetin 3-O-rutinoside (up to 46 folds higher in leaves of integrated 'Clery' compared to fruits), 5-O-caffeoylquinic acid (up to 44 folds higher in leaves of organic 'Duke' compared to fruits), and quercetin (up to 44 folds higher in leaves of integrated 'Bluecrop' when compared to 
fruits). This could be explained with the fact that source-sink imbalance, which can happen with a small sink (demand) combined with a large source capacity (supply), or any other alteration in translocation between source and sink modules, can lead to the accumulation of end-products in leaf photosynthetic tissues $[79,80]$. The accumulation of phenolic compounds in leaves can be clarified with its role as a chemical defense against herbivore insects or some fungi, especially by knowing that quercetin and caffeic acid and its derivates are some of the most important antioxidant stress protectants for plants $[81,82]$. When compared fruits and leaves phenolic profiles, it should be pointed out that epigallocatechin and epicatechin were only found in blueberry fruit samples but not in leaves, while gallocatechin only in blueberry leaves. A flavanone pinocembrin that was just found in strawberry leaves has the potential to treat neurodegenerative diseases, cerebral ischemia, and atherosclerosis [83].

\subsection{Principal Component Analysis}

Principal component analysis (PCA) was used to establish similarity/dissimilarity among the phenolic profiles of blueberry and strawberry fruit and leaf samples that were grown in organic and integrated production systems.

As for fruit samples, PCA was applied on the data matrix 12 (the number of blueberry and strawberry fruit samples) $\times 24$ (quantified phenols, cis, trans-abscisic acid TPC, TAC, and RSA) while using the covariance matrix with autoscaling. The obtained five-component model explained $95.64 \%$ of the total variance. The first two principal components accounted for 61.36 and $15.91 \%$ of total variability, respectively. The PCA score plot (Figure 1A) showed the clustering of investigated fruit samples into two groups along the PC1 axis. As expected, the strawberry fruits were separated from the blueberry fruit samples based on the significant differences in phenolic profiles. From the PCA loading plot, it was possible to identify the most influential variables that were responsible for the clustering (Figure 1B). The presence of syringic acid, sinapic acid, apigenin 8-C-glucoside, 5-O-caffeoylquinic acid, naringenin, quercetin 3-O-rutinoside, epicatechin, and epigallocatechin only in blueberry fruits, together with higher amounts of ferulic acid, caffeic acid, vanillic acid, quercetin 3-O-galactoside, TAC, and TPC, were the most important factors for the discrimination of blueberry samples from the strawberry fruits. On the other hand, the strawberry fruits were grouped based on their higher contents of catechin, kaempferol 3-O-glucoside, cinnamic acid, and $p$-coumaric acid. Interestingly, along with the expected separation of blueberries from strawberries based on a phenol profiles, PCA score plot also discriminated the organically and integrated produced blueberry fruits, and the separation was achieved along PC2. Three organically produced blueberry cultivars were characterized by higher amounts of $p$-hydroxybenzoic acid, quercetin, kaempferol, $p$-coumaric acid, aesculin, ferulic acid, and notably higher values of TPC, TAC, and RSA when compared to same blueberry cultivars that were grown in an integrated production system. Additionally, organically produced blueberry cultivars 'Duke' and 'Nui' stood out from the other blueberry fruits that were based on the highest contents of $p$-hydroxybenzoic acid and quercetin and the highest RSA values. Although organically and integrated strawberry cultivars were not distinguished, the sample 'Alba' grown in organic production system was separated from the other strawberry cultivars due to its higher contents of kaempferol, cinnamic acid, and $p$-coumaric acid.

PCA that was applied on TPC, RSA, and twenty quantified phenolics obtained for blueberry and strawberry leaf samples resulted in five-component model that explained $92.63 \%$ of total variance (PC1, PC2, PC3, PC4, and PC5 accounted for 63.49\%, 13.20\%, 5.81\%, 5.35\%, and 4.79\%, respectively). Based on the PCA correlation plot, there was good discrimination between blueberry and strawberry leaf samples according to PC1 (Figure 2B). Notably higher contents of caffeic acid, kaempferol, 5-O-caffeoylquinic acid, aesculin, kaempferol 3-O-glucoside, $p$-hydroxybenzoic acid, quercetin, and quercetin 3-O-galactoside, as well as the presence of quercetin 3-O-rutinoside, apigenin 8-C-glucoside, and gallocatechin that were exclusively found in blueberry leaf extracts were the most important factors for separation blueberry from strawberry leaf samples. The most influential variables responsible for clustering of strawberry leaf extracts were several phenolic acids (sinapic acid, syringic 
acid, $p$-coumaric acid, and cinnamic acid), catechin, pinocembrin, and RSA (Figure 2B). The PCA score plot (Figure 2A) revealed that leaf samples were not clearly distinguished according to the way of cultivation (organic or integrated). However, one organically produced 'Nui' leaf sample was separated from the other blueberry leaf extracts that were based on its higher contents of the large number of quantified phenols (Table 2).
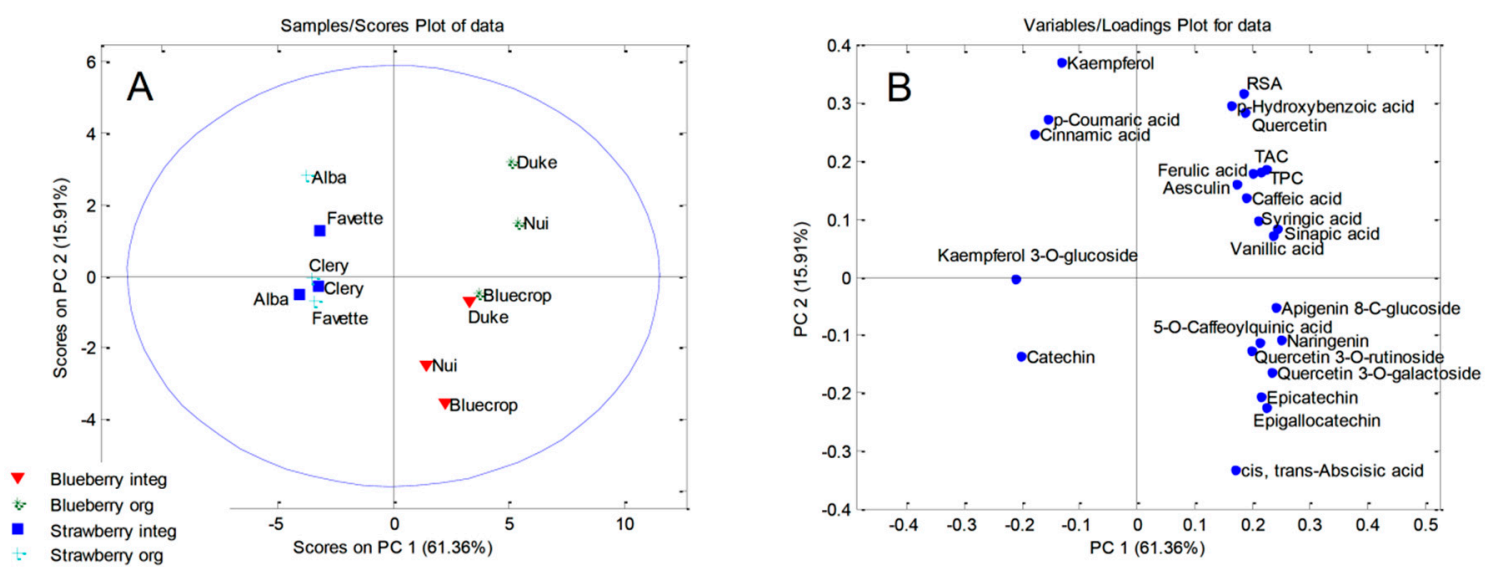

Figure 1. Principal Component Analysis: (A) score plot of the blueberry and strawberry fruit samples; and, (B) loadings plot of the blueberry and strawberry fruit samples.
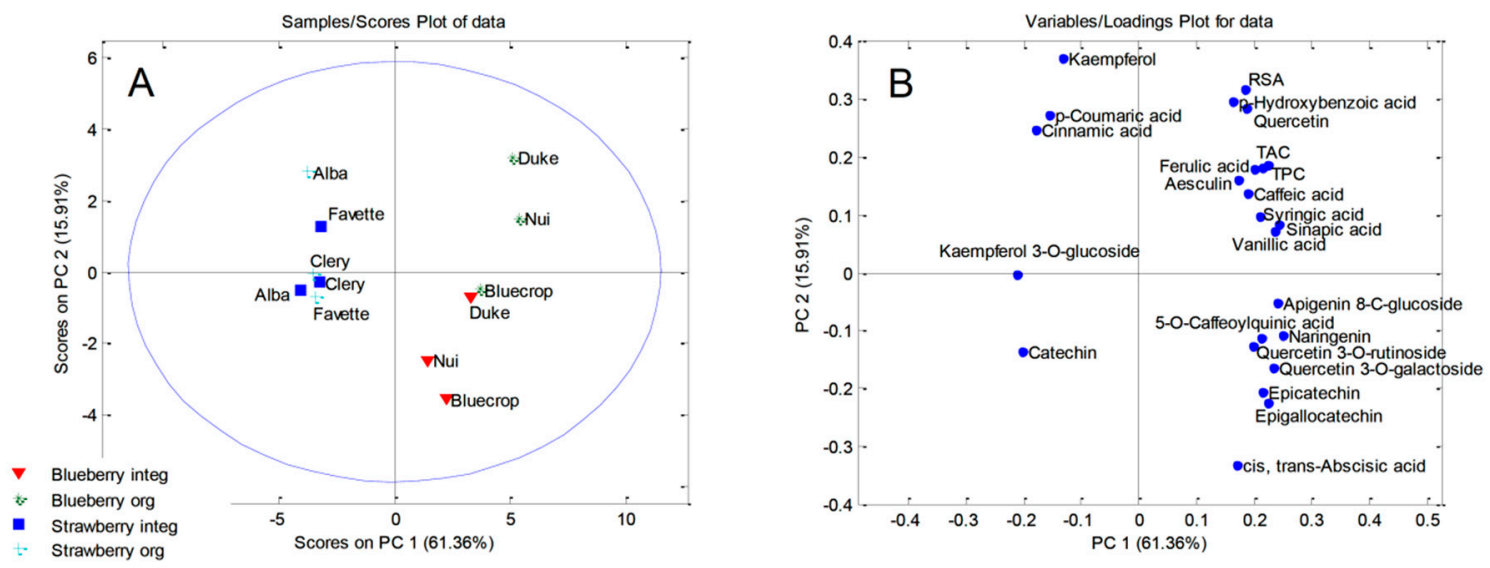

Figure 2. Principal Component Analysis: (A) score plot of the blueberry and strawberry leaf samples; and, (B) loadings plot of the blueberry and strawberry leaf samples.

\section{Materials and Methods}

\subsection{Plant Material}

Both integrated and organic production of three strawberry ('Favette', 'Alba', and 'Clery') and three blueberry ('Bluecrop', 'Duke', and 'Nui') production was organized at the Pambukovica village, municipality $\mathrm{Ub}$, West Serbia. The distance between orchards is $250-300 \mathrm{~m}$, so they represent the same micro-climate zone. Sandy mineral soil is used for establishing strawberry and blueberry orchards. The region has typical continental temperate climatic conditions without any extremes in temperature and rainfall. Integrated production was done according to the Integrated Pest Management Directive 2009/128/ EC, while organic production followed Serbian legislation ['Law on Organic Production' (Official Gazette No. 30/10) and 'The Rulebook on the control and certification of organic production and organic production methods' (Official Gazette No. 48/11)], which fully implemented the EU standards.

Strawberry orchards were planted in July 2013 in double rows on beds that were covered with black polyethylene foil. Plant spacing was $30 \times 30 \mathrm{~cm}$. Blueberry orchards were established with the two-year-old nursery trees that were planted in the spring of 2012 with at a spacing of $3 \mathrm{~m} \times 1 \mathrm{~m}$ 
(3330 bushes ha-1). Fruits picking was done at full maturity in the middle of the harvest in the first year after planting (2014). Water quantity for strawberry irrigation during the most sensitive phases was $40 \mathrm{~m}^{3}$ of water/ha/per application. Blueberry irrigation was done with two laterals with $18 \mathrm{~m}^{3}$ $\left(2 \times 9 \mathrm{~m}^{3}\right.$ of water/ha/per application) during the same growth development. Both fertilization and plant protection in organic and integrated production were done according to the standards that were already explained in our previously published manuscript [1]. In organic strawberry production, powdery mildew and gray mold rot occurred more than in integrated, while, in organic blueberry field, Botrytis blight and fruit rot was more frequent than in organic. The yield losses were around $40 \%$ in both organic strawberry and blueberry production compared to integrate.

The trial was set up in a completely randomized design with three replications and five bushes/plants per replication for each cultivar and in each production system. At harvest, a sample of 20 randomly selected fruits and leaves (from each cultivar/cultivation systems/replication) from all around the bush/plant were taken and used to analyze the sugar profile. Immediately after harvesting, fruits and leaves were placed in hand-fridge, and then carried to the laboratory where they were stored in a freezer at $-20^{\circ} \mathrm{C}$ until chemical analysis.

\subsection{Extraction of Phenols from Fruits and Leaves}

Blueberry and strawberry fruit extracts were prepared according to modified method that was described by Natić et al. [84]. The frozen fruit samples were homogenized and $5 \mathrm{~g}$ of each sample was extracted with $50 \mathrm{~mL}$ of $\mathrm{MeOH}$ acidified with $0.1 \% \mathrm{HCl}$. The extractions were carried during $1 \mathrm{~h}$ (on a magnetic agitator, at room temperature, in dark), in three replications. After each extraction, the extracts were filtered and the clear supernatants were collected. All of the supernatants were evaporated to dryness (reduced pressure at $40^{\circ} \mathrm{C}$ ) while using rotary evaporator IKA RV 8, Staufen, Germany and in residues $\mathrm{MeOH} / \mathrm{H}_{2} \mathrm{O}(60 / 40, v / v)$ solution was added to ca. $50 \mathrm{~mL}$. The extracts were then filtered through $0.45 \mu \mathrm{m}$ membrane filters (Syringe Filter, PTFE, Supelco, Bellefonte, PA, USA).

All leaf samples were washed with water, dried for twenty days (on air, in the dark, at room temperature), and thereafter pooled and ground into a powder. The extraction of phenolics from the leaves was similar to the one described above for fruit, with some modification: about $2 \mathrm{~g}$ of dry leaf samples were extracted with $50 \mathrm{~mL} \mathrm{MeOH} / \mathrm{H}_{2} \mathrm{O}(70: 30, v / v)$ containing $0.1 \% \mathrm{HCl}$ [85].

\subsection{Spectrophotometric Determinations}

Total phenolic content, total anthocyanin content, and radical-scavenging activity were determined while using Folin-Ciocalteu, pH-differential, and DPPH methods, respectively. Pantelić et al. described the methods [86]. All of the measurements were done in triplicate.

\subsection{UHPLC—LTQ Orbitrap $M S^{4}$}

A stock solution of a mixture of phenolics and cis, trans-abscisic acid at a concentration of $1000 \mathrm{ppm}$ was prepared by dissolving standard compounds in methanol. A series of working solutions of concentrations were prepared by diluting the starting solution with the mobile phase: $0.025 ; 0.050$; 0,$100 ; 0.250 ; 0.500 ; 0.750$; and, $1.000 \mathrm{ppm}$. The starting and working solutions were stored in the dark at $4{ }^{\circ} \mathrm{C}$. The calibration curves were obtained by correlating the peak area with the concentration of standard solutions.

The separation of compounds was performed while using ultra-high performance liquid chromatography (UHPLC) system that consisted of an Accela 600 pump and an Accela auto-sampler (Thermo Fisher Scientific, Waltham, MA, USA). The column used for analytical separation was a Syncronis C18 column $(50 \times 2.1 \mathrm{~mm}, 1.7 \mu \mathrm{m}$ particle size, Thermo Fisher Scientific). The mobile phase consists of (A) water with $0.1 \%$ formic acid and (B) acetonitrile with $0.1 \%$ formic acid. The gradient program was as follows: $0.0-1.0 \mathrm{~min} ., 5 \% \mathrm{~B} ; 1.0-14.0 \mathrm{~min} ., 5-95 \% \mathrm{~B} ; 14.0-14.1 \mathrm{~min}$., $95-5 \%$ B; for the next $6 \mathrm{~min} ., 5 \% \mathrm{~B}$. The injection volume for all of the samples was $5 \mu \mathrm{l}$ and the flow rate was $275 \mu \mathrm{L} / \mathrm{min}$. 
The liquid chromatography system was coupled to a linear ion trap-Orbitrap hybrid mass analyzer (LTQ OrbiTrap XL, Waltham, MA, USA). The ionization of compounds was performed in negative mode while using an electron-spray interface (HESI-II, Thermo Fisher Scientific). The ion source parameters were as previously described in Vasić et al. [87]. The mass spectra was recorded in the range of 100 to $1000 \mathrm{~m} / z$. Collision-induced dissociation (CID) was used to study the fragmentation of the tested compounds. The normalized collision energy of the CID was constant $(35 \mathrm{eV})$.

The compounds were quantified according to the exact mass search method ( $\pm 5 \mathrm{ppm})$ based on comparison of retention time and high-resolution accurate mass (HRAM) with that of available reference standards. The results were expressed as $\mathrm{mg} / \mathrm{kg}$. The software ThermoXcalibur 2.2 (Thermo Fisher Scientific, Waltham, MA, USA) and it was employed to process the UHPLC-MS data. The exact masses of the identified compounds that were obtained by high resolution mass spectrometry (HRMS, Waltham, MA, USA) was compared with the exact masses calculated while using Chem Draw software, Waltham, MA, USA. Thus, molecular formulas of unknown compounds were obtained, while their identification was suggested based on specific MS ${ }^{4}$ fragmentation.

\subsection{Statistic Analysis}

All the data were expressed as the mean values of triplicate measurements. Tukey's test was performed to detect the significance of differences $(p \leq 0.05)$ while using the statistical program NCSS (www.ncss.com). Principal Component Analysis was carried out using the PLS_Tool Box software package for MATLAB (Version 7.12.0), Budapest, Hungary. All of the data were group-scaled prior to PCA. The singular value decomposition algorithm (SVD) and a 0.95 confidence level for $\mathrm{Q}$ and Hotelling T2 limits for outliers were chosen.

\section{Conclusions}

High amounts of TPC, TAC, and RSA were detected from the blueberry and strawberry fruit and leaf extracts, which were grown in both an integrated and organic way. The most abundant phenolic compounds in blueberries were 5-O-caffeoylquinic acid, followed by quercetin 3-O-galactoside, while catechin, quercetin, and kaempferol 3-O-glucosid were the most dominant in strawberries. This suggests an importance of the genotype in determining the fruit and leaves composition of bioactive compounds.

The levels of TPC and RSA, and some individual phenolic compounds (caffeic acid, quercetin 3-O-rutinoside, 5-O-caffeoylquinic acid, and quercetin) in leaves as compared to fruits (of both fruit species) were much higher. This indicates that blueberry and strawberry leaves are an excellent source of antioxidants. Having in mind that large amounts of blueberry and strawberry leaves are discarded every year and while considering the high cost associated with growing of this species, the use of their leaves can be advantageous for the producers. Collected leaves could be strongly beneficial, as a high added-value bioactive material for various antioxidant applications in food processing, pharmaceutical, and nutraceutical industry.

Almost all organic fruits and leaves of both fruit species had higher level of TPC; TAC, RSA, and many other phenolic compounds when compared to integrally produced fruits and leaves and it promotes a more nutritious product. It is also very hard to recommend one cultivar, because a genotype with exceptional biochemical content in one production system might poorly perform in another under different agro-ecologic conditions. In temperate climatic conditions of south-east Europe, or similar, fruits of all three studied blueberry cultivars ('Bluecrop', 'Duke', and 'Nui') and strawberry cultivar 'Clery' from organic production, together with leaves of strawberry cultivars 'Alba' and 'Favette' from integrated production, can be a good source of bioactive compounds important from a health perspective.

Supplementary Materials: The following are available online, Figure S1: Proposed structure and fragmentation pathway of compound 37, Table S1: Presence of each identified compound in integrated and organic strawberry 
and blueberry fruit samples, Table S2: Presence of each identified compound in integrated and organic strawberry and blueberry leaf samples.

Author Contributions: Conceptualization, M.F.A. and M.M.; methodology and writing, M.F.A. and M.N.; validation and investigation, M.F.A.; formal analysis, U.G., M.S. and D.D.Z.; writing, review and editing, M.F.A., J.M. and M.M.

Funding: This research received no external funding.

Acknowledgments: This study was supported by the Ministry of Education, Science and Technological Development of the Republic of Serbia (projects No. 172017 and III 46008) and The Research Council of Norway (grant number 280376).

Conflicts of Interest: The authors declare no conflict of interest.

\section{References}

1. Fotirić Akšić, M.; Tosti, T.; Sredojević, M.; Milivojević, J.; Meland, M.; Natić, M. Comparison of sugar profile between leaves and fruits of blueberry and strawberry cultivars grown in organic and integrated production system. Plants 2019, 8, 205. [CrossRef] [PubMed]

2. Vittori, L.D.; Mazzoni, L.; Battino, M.; Mezzetti, B. Pre-harvest factors influencing the quality of berries. Sci. Hortic. Amsterdam 2018, 233, 310-322. [CrossRef]

3. Milivojević, J.; Radivojevic, D.; Ruml, M.; Dimitrijevic, M.; Dragisic Maksimovic, J. Does microclimate under grey colored hail protection net affect biological and nutritional properties of 'Duke' highbush blueberry (V. corymbosum L.)? Fruits 2016, 71, 161-170. [CrossRef]

4. Giampieri, F.; Tulipani, S.; Alvarez-Suarez, J.M.; Quiles, J.L.; Mezzetti, B.; Battino, M. The strawberry: Composition, nutritional quality, and impact on human health. Nutrition 2012, 28,9-19. [CrossRef] [PubMed]

5. Kizos, T.; Veikontis, G.; Marín-Guirao, J.I. Comparison of organic and integrated farming systems: The case of sultana table grapes in Korinthos, Greece. J. Sustain. Agric. 2011, 35, 27-47. [CrossRef]

6. Stockdale, E.A.; Lampkin, N.H.; Hovi, M.; Keatinge, R.; Lennartsson, E.K.M.; Macdonald, D.W.; Padel, S.; Tattersall, F.H.; Wolfe, M.S.; Watson, C.A. Agronomic and environmental implications of organic farming systems. Adv. Agron. 2001, 70, 261-327.

7. Ochmian, I.; Kozos, K.; Chelpiñski, P.; Szczepanek, M. Comparison of berry quality in highbush blueberry cultivars grown according to conventional and organic methods. Turk. J. Agric. For. 2015, 39, 174-181. [CrossRef]

8. Granatstein, D.; Kirby, E.; Willer, H. Global area and trends of organic fruit production. Acta Hortic. 2013, 1001, 383-394. [CrossRef]

9. The World of Organic Agriculture. Statistics and Emerging Trends. Available online: https://shop.fibl.org/ CHen/mwdownloads/download/link/id/1202/?ref=1 (accessed on 29 October 2019).

10. You, Q.; Wang, B.; Chen, F.; Huang, Z.; Wang, X.; Luo, P.G. Comparison of anthocyanins and phenolics in organically and conventionally grown blueberries in selected cultivars. Food Chem. 2011, 125, 201-208. [CrossRef]

11. Olsson, M.E.; Andersson, C.S.; Oredsson, S.; Berglund, R.H.; Gustavsson, K. Antioxidant levels and inhibition of cancer cell proliferation in vitro by extracts from organically and conventionally cultivated strawberries. J. Agric. Food Chem. 2006, 54, 1248-1255. [CrossRef]

12. Brandt, K.; Mølgaard, J.P. Organic agriculture: Does it enhance or reduce the nutritional value of plant foods. J. Sci. Food Agric. 2001, 81, 924-931. [CrossRef]

13. Nile, S.H.; Park, S.W. Edible berries: Bioactive components and their effect on human health. Nutrition 2014, 30, 134-144. [CrossRef] [PubMed]

14. Milivojević, J.; Rakonjac, V.; Fotirić Akšić, M.; Bogdanović Pristov, J.; Maksimović, V. Classification and fingerprinting of different berries based on biochemical profiling and antioxidant capacity. Pesqui. Agropecu. Bras. 2013, 48, 1285-1294. [CrossRef]

15. Kalt, W.; Lawand, C.; Ryan, D.; McDonald, J.E.; Donner, H. Oxygen radical absorbing capacity, anthocyanin and phenolic content of highbush blueberries (Vaccinium corymbosum, L.), during ripening and storage. J. Am. Soc. Hortic. Sci. 2003, 128, 917-923. [CrossRef]

16. Skrovankova, S.; Sumczynski, D.; Mlcek, J.; Jurikova, T.; Sochor, J. Bioactive compounds and antioxidant activity in different types of berries. Int. J. Mol. Sci. 2015, 16, 24673-24706. [CrossRef] 
17. Calò, R.; Marabini, L. Protective effect of Vaccinium myrtillus extract against UVA- and UVB-induced damage in a human keratinocyte cell line (HaCaT cells). J. Photochem. Photobiol. B. 2014, 132, 27-35. [CrossRef]

18. Milivojević, J.; Maksimović, V.; Dragišić Maksimović, J.; Radivojević, D.; Poledica, M.; Ercişli, S. A comparison of major taste- and health-related compounds of Vaccinium berries. Turkish, J. Biol. 2012, 36, 738-745.

19. Rostamian, V.; Shakeri, F.; Estakhr, J. The effect of hydro-alcoholic extract of strawberry leaf on sugar and lipids in serum of diabetic rats. Pharmacology Online 2011, 3, 1171-1175.

20. Mandave, P.; Rani, S.; Kuvalekar, A.; Ranjekar, P. Antiglycation, antioxidant and antidiabetic activity of mature strawberry (Fragaria x ananassa) fruts. Int. J. Appl. Biol. Pharm. 2013, 4, 168-177.

21. Routray, W.; Orsat, V. MAE of phenolic compounds from blueberry leaves and comparison with other extraction methods. Ind. Crop. Prod. 2014, 58, 36-45. [CrossRef]

22. Yuji, K.; Sakaida, H.; Kai, T.; Fukuda, N.; Yukizaki, C.; Sakai, M.; Tsubouchi, H.; Kataoka, H. Effect of dietary blueberry (Vaccinium ashei Reade) leaves on serum and hepatic lipid levels in rats. J. Oleo Sci. 2013, 62, 89-96. [CrossRef] [PubMed]

23. Vyas, P.; Kalidindi, S.; Chibrikova, L.; Igamberdiev, A.U.; Weber, J.T. Chemical analysis and effect of blueberry and lingonberry fruits and leaves against glutamate-mediated excitotoxicity. J. Agric. Food Chem. 2013, 61, 7769-7776. [CrossRef] [PubMed]

24. Sakaida, H.; Nagao, K.; Higa, K.; Shirouchi, B.; Inoue, N.; Hidaka, F.; Kai, T.; Yanagita, T. Effect of Vaccinium ashei Reade leaves on angiotensin converting enzyme activity in vitro and on systolic blood pressure of spontaneously hypertensive rats In Vivo. Biosci. Biotechnol. Biochem. 2007, 71, 2335-2337. [CrossRef] [PubMed]

25. Martineau, L.C.; Couture, A.; Spoor, D.; Benhaddou-Andaloussi, A.; Harris, C.; Meddah, B.; Leduc, C.; Burt, A.; Vuong, T.; Le Mai, P.; et al. Anti-diabetic properties of the Canadian low bush blueberry Vaccinium angustifolium Ait. Phytomedicine 2006, 13, 612-623. [CrossRef] [PubMed]

26. Naglaa, M.; Saleh, A. Influence of ethanolic extract of strawberry leaves for abrogating bromate hazards in male rats. J. Basic Appl. Zool. 2019, 80, 1-19.

27. Ibrahim, D.S.; Abd El-Maksoud, M.A.E. Effect of strawberry (Fragaria ananassa) leaf extract on diabetic nephropathy in rats. Int. J. Exp. Pathol. 2015, 96, 87-93. [CrossRef]

28. Kårlund, A.; Salminen, J.P.; Koskinen, P.; Ahern, J.R.; Karonen, M.; Tiilikkala, K.; Karjalainen, R.O. Polyphenols in strawberry (Fragaria x ananassa) leaves induced by plant activators. J. Agric. Food Chem. 2014, 62, 4592-4600. [CrossRef]

29. Okan, O.T.; Deniz, I.; Yayli, N.; Sat, I.G.; Öz, M.; Hatipo־gluSerdar, G. Antioxidant activity, sugar content and phenolic profiling of blueberries cultivars: A comprehensive. Not. Bot. Horti. Agrobo. 2018, 46, 639-652. [CrossRef]

30. Mezzetti, B.; Balducci, F.; Capocasa, F.; Cappelletti, R.; Di Vittori, L.; Mazzoni, L.; Giampieri, F.; Battino, M. Can we breed a healthier strawberry and claim it? Acta Hortic. 2016, 1117, 7-14. [CrossRef]

31. Capocasa, F.; Scalzo, J.; Mezzetti, B.; Battino, M. Combining quality and antioxidant attributes in the strawberry: The role of genotype. Food Chem. 2008, 111, 872-878. [CrossRef]

32. Wang, S.; Chen, C.-T.; Sciarappa, W.; Wang, C.; Camp, M. Fruit quality, antioxidant capacity and flavonoid content of organically and conventionally grown blueberries. J. Agric. Food Chem. 2008, 56, 5788-5794. [CrossRef] [PubMed]

33. Aaby, K.; Ekeberg, D.; Skrede, G. Characterization of phenolic compounds in strawberry (Fragaria ananassa) fruits by different HPLC detectors and contribution of individual compounds to total antioxidant capacity. J. Agric. Food Chem. 2007, 55, 4395-4406. [CrossRef] [PubMed]

34. Motilva, M.J.; Serra, A.; Macià, A. Analysis of food polyphenols by ultra high-performance liquid chromatography coupled to mass spectrometry: An overview. J. Chromatogr. A 2013, 1292, 66-82. [CrossRef] [PubMed]

35. Castrejớn, A.D.R.; Eichholz, I.; Rohn, S.; Kroh, L.W.; Huyskens-Keil, S. Phenolic profile and antioxidant activity of highbush blueberry (Vaccinium corymbosum, L.) during fruit maturation and ripening. Food Chem. 2008, 109, 564-572. [CrossRef]

36. Mikulic Petkovsek, M.; Stampar, F.; Veberic, R. The effect of prohexadione-calcium on the phenolic content in developing fruits and leaves of apple trees. J. Food Agric. Environ. 2009, 7, 369-375. 
37. Kitano, M.; Araki, T. Environmental effects on dynamics of fruit growth and photo assimilate translocation in tomato plants (2)-Analysis of phloem sap and xylem sap fluxes and fruit water balance. Environ. Control Biol. 2001, 39, 43-51. [CrossRef]

38. Dragišić Maksimović, J.; Poledica, M.; Mutavdžić, D.; Mojović, M.; Radivojević, D.; Milivojević, J. Variation in nutritional quality and chemical composition of fresh strawberry fruit: Combined effect of cultivar and storage. Plant Foods Hum. Nutr. 2015, 70, 77-84. [CrossRef]

39. Connor, A.M.; Luby, J.J.; Tong, C.B.S.; Finn, C.E.; Hancock, J.F. Variation and heritability estimates for antioxidant activity. Total phenolic content and anthocyanin content in blueberry progenies. J. Am. Soc. Hortic. Sci. 2002, 1, 82-88. [CrossRef]

40. Jin, P.; Wang, S.Y.; Wang, C.Y.; Zheng, Y. Effect of cultural system and storage temperature on antioxidant capacity and phenolic compounds in strawberries. Food Chem. 2011, 124, 262-270. [CrossRef]

41. Prodorutti, D.; Pertot, I.; Giongo, L.; Gessler, C. Highbush blueberry: Cultivation, protection, breeding and biotechnology. Eur. J. Plant Sci. Biotechnol. 2007, 1, 44-56.

42. Häkkinen, S.; Törrönen, A.R. Content of flavonols and selected phenolic acids in strawberries and Vaccinium species: Influence of cultivar, cultivation site and technique. Food Res. Int. 2000, 33, 517-524. [CrossRef]

43. Tomić, J.; Milivojević, J.; Pešaković, M. The response to bacterial inoculation is cultivar-related in strawberries. Turk. J. Agric. For. 2015, 39, 332-334. [CrossRef]

44. Weibel, F.P.; Bickel, R.; Leuthold, S.; Alfoeldi, T.; Niggli, U. Are organically grown apples tastier and healthier? A comparative field study using conventional and alternative methods to measure fruit quality. Acta Hortic. 2000, 517, 417-426. [CrossRef]

45. Panico, A.M.; Garufi, F.; Nitto, S.; Di Mauro, R.; Longhitano, R.C.; Magrı, G.; Catalfo, A.; Serrentino, M.E.; De Guidi, G. Antioxidant activity and phenolic content of strawberry genotypes from Fragaria $x$ ananassa. Pharm. Biol. 2009, 47, 203-208. [CrossRef]

46. Crespo, P.; Bordonaba, J.G.; Terry, L.A.; Carlen, C. Characterization of major taste and health-related compounds of four strawberry genotypes grown at different Swiss production sites. Food Chem. 2010, 122, 16-24. [CrossRef]

47. Stevenson, D.; Scalzo, J. Anthocyanin composition and content of blueberries from around the world. J. Berry Res. 2012, 2, 179-189.

48. Cezarotto, V.S.; Giacomelli, S.R.; Vendruscolo, M.H.; Vestena, A.S.; Cezarotto, C.S.; Cruz, R.C.; Maurer, L.H.; Ferreira, L.M.; Emanuelli, T.; Cruz, L. Influence of harvest season and cultivar on the variation of phenolic compounds composition and antioxidant properties in Vaccinium ashei Leaves. Molecules 2017, 22, 1603. [CrossRef]

49. Wu, H.; Chai, Z.; Hutabarat, R.P.; Zeng, Q.; Niu, L.; Li, D.; Yu, H.; Huang, W. Blueberry leaves from 73 different cultivars in southeastern China as nutraceutical supplements rich in antioxidants. Food Res. Int. 2019, 122, 548-560. [CrossRef]

50. Grace, M.; Xiong, J.; Esposito, D.; Ehlenfeldt, M.; Lila, M. Simultaneous LC-MS quantification of anthocyanins and non-anthocyanin phenolics from blueberries with widely divergent profiles and biological activities. Food Chem. 2019, 227, 336-346. [CrossRef]

51. Buřičová, L.; Andjelkovic, M.; Čermáková, A.; Réblová, Z.; Jurček, O.; Kolehmainen, E.; Verhé, R.; Kvasnička, F. Antioxidant capacity and antioxidants of strawberry, blackberry, and raspberry leaves. Czech, J. Food Sci. 2011, 29, 181-189. [CrossRef]

52. Ryan, J.J. Flavonol glycosides of cultivated strawberry. J. Food Sci. 1971, 36, 867-870. [CrossRef]

53. Ayaz, F.A.; Hayirlioglu-Ayaz, S.; Gruz, J.; Novak, O.; Strnad, M. Separation, Characterization, and Quantitation of Phenolic Acids in a Little-Known Blueberry (Vaccinium arctostaphylos L.) Fruit by HPLC-MS. J. Agric. Food Chem. 2005, 53, 8116-8122. [CrossRef] [PubMed]

54. La Barbera, G.; Capriotti, A.L.; Cavaliere, C.; Piovesana, S.; Samperi, R.; Chiozzi, R.Z.; Laganà, A. Comprehensive polyphenol profiling of a strawberry extract (Fragaria $x$ ananassa) by ultra-high-performance liquid chromatography coupled with high-resolution mass spectrometry. Anal. Bioanal. Chem. 2017, 409, 2127-2142. [CrossRef] [PubMed]

55. Ablajan, K.; Abliz, A.; Shang, X.Y.; He, J.M.; Rui- Ping Zhang, R.P.; Shi, J.G. Structural characterization of flavonol 3,7- di-O glycosides and determination of the glycosylation position by using negative ion electrospray ionisation tandem mass spectrometry. J. Mass Spectrom. 2006, 41, 352-360. [CrossRef] 
56. Cuyckens, F.; Claeys, M. Determination of the glycosylation site in flavonoid mono-O-glycosides by collision-induced dissociation of electrospray-generated deprotonated and sodiated molecules. J. Mass Spectrom. 2005, 40, 364-372. [CrossRef]

57. Mondolot, L.; La Fisca, P.; Buatois, B.; Talansier, E.; De Kochko, A.; Campa, C. Evolution in caffeoylquinic acid content and histolocalization during Coffea canephora leaf development. Ann. Bot. 2006, 98, 33-40. [CrossRef]

58. Wang, L.; Yue, Z.; Guo, M.; Fang, L.; Bai, L.; Li, X.; Tao, Y.; Wang, S.; Liu, Q.; Zhi, D.; et al. Dietary flavonoid hyperoside induces apoptosis of activated human LX-2 hepatic stellate cell by suppressing canonical NF- $\mathrm{KB}$ signaling. Biomed. Res. Int. 2016, 2016, 1068528. [CrossRef]

59. Zimmer, K.R.; Blum-Silva, C.H.; Souza, A.L.K.; Wulffschuch, M.; Reginatto, H.F.; Pereira, C.M.P.; Macedo, A.J.; Lencina, L.C. The antibiofilm effect of blueberry fruit cultivars against Staphylococcus epidermidis and Pseudomonas aeruginosa. J. Med. Food 2014, 17, 324-331. [CrossRef]

60. Yang, G.; Yue, J.; Gong, X.; Qian, B.; Wang, H.; Deng, Y.; Zhao, Y. Blueberry leaf extracts incorporated chitosan coatings for preserving postharvest quality of fresh blueberries. Postharvest Biol. Technol. 2014, 92, 46-53. [CrossRef]

61. Howard, L.R.; Clark, J.R.; Brownmiller, C. Antioxidant capacity and phenolic content in blueberries as affected by genotype and growing season. J. Sci. Food Agric. 2003, 83, 1238-1247. [CrossRef]

62. Wang, S.Y.; Millner, P. Effect of different cultural systems on antioxidant capacity, phenolic content, and fruit quality of strawberries (Fragaria x aranassaDuch.). J. Agric. Food Chem. 2009, 57, 9651-9657. [CrossRef]

63. Sultana, B.; Anwar, F. Flavonols (kaempeferol, quercetin, myricetin) contents of selected fruits, vegetables and medicinal plants. Food Chem. 2008, 108, 879-884. [CrossRef]

64. Skupień, K.; Oszmianski, J.; Kostrzewa-Nowak, D.; Tarasiuk, J. In vitro anti-leukemic activity of extracts from berry plant leaves against sensitive and multidrug resistant HL60 cells. Cancer Lett. 2006, 236, $282-291$. [CrossRef]

65. Harris, C.S.; Burt, A.J.; Saleem, A.; Le, P.M.; Martineau, L.C.; Haddad, P.S.; Bennett, S.A.; Arnason, J.T. A single HPLC-PAD-APCI/MS method for the quantitative comparison of phenolic compounds found in leaf, stem, root and and fruit extracts of Vaccinium angustifolium. Phytochem. Anal. 2007, 18, 161-169. [CrossRef]

66. Oszmianski, J.; Wojdylo, A.; Gorzelany, J.; Kapusta, I. Identification and characterization of low molecular weight polyphenols in berry leaf extracts by HPLC-DAD and LC-ESI/MS. J. Agric. Food Chem. 2011, 59, 12830-12835. [CrossRef]

67. Li, C.; Feng, J.; Huang, W.-Y.; An, X.-T. Composition of polyphenols and antioxidant activity of rabbiteye blueberry (Vaccinium ashei) in Nanjing. J. Agric. Food Chem. 2013, 61, 523-531. [CrossRef]

68. Kaur, T.; Bhat, H.A.; Bhat, R.; Kumar, A.; Bindu, K.; Koul, S.; Vyas, D. Physio-chemical and antioxidant profiling of Salvia sclarea L. at different climates in north-western Himalayas. Acta Physiol. Plant. 2015, 37, 132. [CrossRef]

69. Cheel, J.; Tumova, L.; Areche, C.; Van Antwerpen, P.; Neve, J.; Zouaoui-Boudjeltia, K.; Martin, A.S.; Vokral, I.; Wsol, V.; Neugebauerova, J. Variations in the chemical profile and biological activities of licorice (Glycyrrhiza glabra L.), as influenced by harvest times. Acta Physiol. Plant. 2013, 35, 1337-1349. [CrossRef]

70. Wasilewska, A.; Vlad, F.; Sirichandra, C.; Redko, Y.; Jammes, F.; Valon, C.; Frei dit Frey, N.; Leung, J. An update on abscisic acid signaling in plants and more. Mol. Plant 2008, 1, 198-217. [CrossRef]

71. Taiz, L.; Zeiger, E. Abscisic acid: A seed maturation and anti stress signal. In Plant Physiology; Sinauer Associates, Inc.: Sunderland, MA, USA, 2006; pp. 423-558.

72. Li, C.; Jia, H.; Chai, Y.; Shen, Y. Abscisic acid perception and signaling transduction in strawberry: A model for non-climacteric fruit ripening. Plant Signal. Behav. 2011, 6, 1950-1953. [CrossRef]

73. Symons, G.M.; Chua, Y.-J.; Ross, J.J.; Quittenden, L.J.; Davies, N.W.; Reid, J.B. Hormonal changes during non-climacteric ripening in strawberry. J. Exp. Bot. 2012, 63, 4741-4750. [CrossRef] [PubMed]

74. Karppinen, K.; Tegelberg, P.; Häggman, H.; Jaakola, L. Abscisic acid regulates anthocyanin biosynthesis and gene expression associated with cell wall modification in ripening bilberry (Vaccinium myrtillus L.) fruits. Front. Plant Sci. 2018, 9, 1259. [CrossRef] [PubMed]

75. Setha, S. Roles of abscisic acid in fruit ripening. Walailak J. Sci. Tech. 2012, 9, 297-308.

76. Srivastava, L.M. Plant Growth and Development. Hormones and Environment, 1st ed.; Oxford Academic Press: Cambridge, MA, USA, 2002; pp. 1-772.

77. Harris, J. Abscisic acid: Hidden architect of root system structure. Plants 2015, 4, 548-572. [CrossRef] [PubMed] 
78. Ehlenfeldt, M.K.; Prior, R.L. Oxygen radical absorbance capacity (ORAC) and phenolic and anthocyanin concentrations in fruit and leaf tissues of highbush blueberry. J. Agric. Food Chem. 2001, 49, 2222-2227. [CrossRef]

79. White, A.C.; Rogers, A.; Rees, M.; Osborne, C.P. How can we make plants grow faster? A source-sink perspective on growth rate. J. Exp. Bot. 2016, 67, 31-45. [CrossRef]

80. Ruiz-Vera, U.; De Souza, A.; Long, S.; Ort, D. The role of sink strength and nitrogen availability in the down-regulation of photosynthetic capacity in field-grown Nicotiana tabacum L. at elevated $\mathrm{CO}_{2}$ concentration. Front. Plant Sci. 2017, 8, 998. [CrossRef]

81. Kurepa, J.; Shull, T.E.; Smalle, J.A. Quercetin feeding protects plants against oxidative stress. F1000Research 2016, 5, 2430. [CrossRef]

82. Riaz, U.; Kharal, M.A.; Murtaza, G.; Zaman, Q.; Javaid, S.; Malik, H.A.; Aziz, H.; Abbas, Z. Prospective roles and mechanisms of caffeic acid in counter plant stress: A mini review. Pakistan J. Agri. Res. 2019, 32, 8-19. [CrossRef]

83. Lan, X.; Wang, W.; Li, Q.; Wang, J. The natural flavonoid pinocembrin: Molecular targets and potential therapeutic applications. Mol. Neurobiol. 2016, 53, 1794-1801. [CrossRef]

84. Natić, M.; Dabić, D.; Papetti, A.; FotirićAkšić, M.; Ognjanov, V.; Ljubojević, M.; Tešić, Ž. Analysis and characterisation of phytochemicals in mulberry (Morus alba L.) fruits grown in Vojvodina, North Serbia. Food Chem. 2015, 171, 128-136. [CrossRef] [PubMed]

85. Pantelić, M.; Dabić Zagorac, D.; Ćirić, I.; Pergal, M.; Relić, D.; Todić, S.; Natić, M. Phenolic profiles, antioxidant activity and minerals in leaves of different grapevine varieties grown in Serbia. J. Food Compos. Anal. 2017, 62, 76-83. [CrossRef]

86. Pantelić, M.; DabićZagorac, D.; Natić, M.; Gašić, U.; Jović, S.; Vujović, D.; Popović-Djordjević, J. Impact of clonal variability on phenolics and radical scavenging activity of grapes and wines: A study on the recently developed Merlot and Cabernet Franc clones (Vitis vinifera L.). PLoS ONE 2016, 11, e0163823.

87. Vasić, V.; Gašić, U.; Stanković, D.; Lušić, D.; Vukić-Lušić, D.; Milojković-Opsenica, D.; Tešić, Ž. Towards better quality criteria of European honeydew honey: Phenolic profile and antioxidant capacity. Food Chem. 2019, 274, 629-641. [CrossRef] [PubMed]

Sample Availability: Samples of the compounds are not available from the authors.

(C) 2019 by the authors. Licensee MDPI, Basel, Switzerland. This article is an open access article distributed under the terms and conditions of the Creative Commons Attribution (CC BY) license (http://creativecommons.org/licenses/by/4.0/). 